\title{
The mass-loss rates of red supergiants and the de Jager prescription ${ }^{\star}$
}

\begin{abstract}
N. Mauron and E. Josselin
GRAAL, CNRS and Université Montpellier II, Place Bataillon, 34095 Montpellier, France

e-mail: nicolas . mauron@univ-montp2 . fr

Received 5 January 2010 / Accepted 24 October 2010

ABSTRACT

Mass loss of red supergiants (RSG) is important for the evolution of massive stars, but is not fully explained. Several empirical prescriptions have been proposed, trying to express the mass-loss rate $(\dot{M})$ as a function of fundamental stellar parameters (mass, luminosity, effective temperature). Our goal is to test whether the de Jager et al. (1988, A\&AS, 72, 259) prescription, which is used in some stellar evolution models, is still valid in view of more recent mass-loss determinations. By considering 40 Galactic RSGs with an infrared excess and an IRAS 60- $\mu \mathrm{m}$ flux larger than $2 \mathrm{Jy}$, and assuming a gas-to-dust mass ratio of 200, we find that the de Jager rate agrees within a factor 4 with most $\dot{M}$ estimates based on the $60-\mu \mathrm{m}$ signal. It also agrees with six of the only eight Galactic RSGs for which $\dot{M}$ can be measured more directly through observations of the circumstellar gas. The two objects that do not follow the de Jager prescription (by an order of magnitude) are $\mu$ Cep and NML Cyg. We also considered the RSGs of the Magellanic Clouds. Thanks to the results of previous research, we find that the RSGs of the Small Magellanic Cloud have mass-loss rates consistent with the de Jager rate scaled by $\left(Z / Z_{\odot}\right)^{\alpha}$, where $Z$ is the metallicity and $\alpha$ is 0.7 . The situation is less clear for the RSGs of the Large Magellanic Cloud. In particular, for $L>1.6 \times 10^{5} L_{\odot}$, one finds numerous RSGs (except WOH-G64) with $\dot{M}$ significantly smaller than the de Jager rate and indicating that $\dot{M}$ would no longer increase with $L$. Before this odd situation is confirmed through further analyses of LMC RSGs, we suggest to keep the de Jager prescription unchanged at solar metallicity in the stellar evolutionary models and to apply a $\left(Z / Z_{\odot}\right)^{0.7}$ dependence.
\end{abstract}

Key words. stars: massive - stars: mass-loss - supergiants

\section{Introduction}

The red supergiant stars (hereafter RSG) are massive stars evolving beyond the main sequence and passing a fraction of their lives in the cool upper region of the Hertzsprung-Russell (H-R) luminosity-temperature diagram. In the solar neighbourhood, the initial mass of RSGs is about 8 to $25 M_{\odot}$, their effective temperature is for most objects 3000 to $4000 \mathrm{~K}$, and their luminosity is between $2 \times 10^{4}$ and $\sim 6 \times 10^{5} L_{\odot}$. Several important works have recently revisited the basic properties of these stars in the Galaxy and in other systems like the Magellanic Clouds and M31 (Massey 1998; Levesque et al. 2005, hereafter LM05; Massey et al. 2008; Levesque 2009, and references therein). In general, a fairly good agreement between recent observations and the predictions of evolutionary models has been established. However, one of the important processes occuring at the surface of these stars, mass loss, is still poorly known. The radii of RSGs are huge, typically $500-1500 R_{\odot}$. Consequently, the gravity of these stars is very small, about 50000 times smaller than that of the Sun. The small gravity favours ejection of matter to the interstellar medium, and indeed some RSGs lose mass at a high rate, $10^{-6}$ to $10^{-4} M_{\odot} \mathrm{yr}^{-1}$.

Mass loss has several consequences. First, a significant fraction of the stellar mass is lost, which is important for the subsequent evolution of the star. Secondly, the ejected circumstellar matter enriches the ISM in dust, e.g. diverse types of silicates (see for example Verhoelst et al. 2009) and even carbonaceous

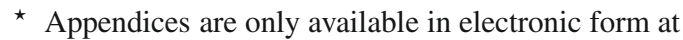
http: //www . aanda.org matter (Sylvester et al. 1994, 1998). One can note also that some types of core-collapse supernovae have RSG progenitors (Smartt et al. 2008; Josselin \& Lançon 2009), so that the density and radial distribution of the RSG winds play an important role in the expansion and evolution of the supernova remnant (Chevalier et al. 2006; Smith et al. 2009; van Loon 2010).

Despite the importance of RSG mass loss, a quantitative physical model of the winds has yet to be established. Like for other mass-losing cool giants, atmospheric motions such as pulsation, convection, and shocks occur (e.g. Kiss et al. 2006; Josselin \& Plez 2007), and this may produce out-moving gas sufficiently cool for dust to form. Then, radiation pressure on the grains may be sufficient to drive the outflow (see for example Habing et al. 1994). Although this scenario is plausible, some important details are still unclear. For example, the condensation of the Fe-rich silicate grains, which are crucial to drive the wind, is not well understood (Woitke 2007). When dust is incompletely formed or absent, other mass-loss mechanisms have to be found, but none is completely satisfactory (e.g. Bennett 2010, and references therein).

Because no physical model exists, diverse empirical parametrizations are used in stellar evolution codes for estimating $\dot{M}$ as a function of basic stellar characteristics, i.e. mass, luminosity, and effective temperature. An important example is the empirical law built by de Jager et al. (1988, hereafter dJ88). This law was based on observational estimates of $\dot{M}$ for stars located over the whole H-R diagram, including 15 RSGs. It is used in the Cambridge stellar evolution code with a $\left(Z / Z_{\odot}\right)^{0.5}$ metallicity scaling (Stancliffe \& Eldridge 2009). The dJ88 law was also used 
in past evolution models of the Geneva group (described in, e.g., Maeder \& Meynet 1989; Eggenberger et al. 2008). However, in the most recent Geneva code, the dJ88 prescription is used only for yellow supergiants with $T_{\text {eff }}$ between $5000 \mathrm{~K}$ and $8000 \mathrm{~K}$. For smaller $T_{\text {eff }}$, a power-law in $L, \dot{M} \propto L^{1.7}$, is adopted with no metallicity scaling.

The goal of this paper is to investigate whether this de Jager formulation still agrees with more recently obtained mass-loss rates. For example, mass-loss rates of RSGs were estimated with IRAS observations of the circumstellar dust (Jura \& Kleinman 1990, hereafter JK90; Josselin et al. 2000, hereafter JB00) or from ISO spectra (Verhoelst et al. 2009). Several radio millimeter observations of CO from RSGs have also been made since 1988 (JB00; Woodhams 1993). The question is whether these more recent measurements support the $\mathrm{dJ} 88$ prescription.

In the following, Sect. 2 recalls the prescription of de Jager and compares it with a few others. In Sect. 3 we compare the dJ88 rate with measured mass-loss rates of RSGs, and we consider three different cases: i) the RSGs of the solar neighbourhood and $\dot{M}$ estimated with their infrared excess at $60 \mu \mathrm{m}$; ii) the eight cases of these RSGs for which circumstellar gas has been directly observed and used to derive $\dot{M}$; iii) the RSGs of the Magellanic Clouds. In Sect. 4 we discuss our results and conclude in Sect. 5 .

\section{Comparison of de Jager rates with other prescriptions}

In the paper of $\mathrm{dJ} 88$, the authors collected a large number of mass-loss rates determined for 271 Galactic stars located over the whole H-R diagram. The mass-loss rates could be matched by a single empirical formula in which the parameters are the effective temperature $T_{\text {eff }}$ and the luminosity $L$. Hereafter, we call this formula "the de Jager rate". According to dJ88, the accuracy of this formula is about 0.45 in $\log \dot{M}$, i.e. about a factor of 3. Because the de Jager formula comprises 20 Chebycheff polynomia, the dependence of $\dot{M}$ on $T_{\text {eff }}$ and $L$ cannot be seen clearly. Therefore, we show in Fig. 1 the situation for RSGs. The de Jager rate is plotted for two different effective temperatures, 3500 and $4000 \mathrm{~K}$, since the majority of Galactic RSGs have their $T_{\text {eff }}$ between these values (LM05). More precisely, in the list of 74 RSGs of LM05 (with a M-type classification), only $\alpha$ Her has a lower temperature, with $3450 \mathrm{~K}$, but it is not a massive star, $M \approx 2.1 M_{\odot}$ according to Schröder \& Cuntz (2007). Figure 1 shows that $\dot{M}$ is not very dependent on $T_{\text {eff }}$ in the de Jager formulation, and that $\dot{M}$ only depends on $L$.

Among the 271 stars (hot or cool, luminous or not) that are at the basis for the dJ88 prescription, there are only 15 Galactic RSGs, for which dJ88 compiled $T_{\text {eff }}, L$ and an observational estimate of $\dot{M}$, or an average of estimates. These compiled data are listed in Appendix A together with results of this work, which we discuss below. It can be noted that the $T_{\text {eff }}$ are significantly cooler than those recently determined by LM05, when available. In addition, about half of the estimated $\dot{M}$ are based on the early paper of Gehrz \& Woolf (1971). So, we think it appropriate to reexamine the validity of the $\mathrm{dJ} 88$ prescription with a larger sample of objects (40 are considered in this work) and more recent data.

There exists a second formulation published later by Nieuwenhuijzen and de Jager (1990, hereafter NJ90). The stellar mass is taken into account, and the dependence of $\dot{M}$

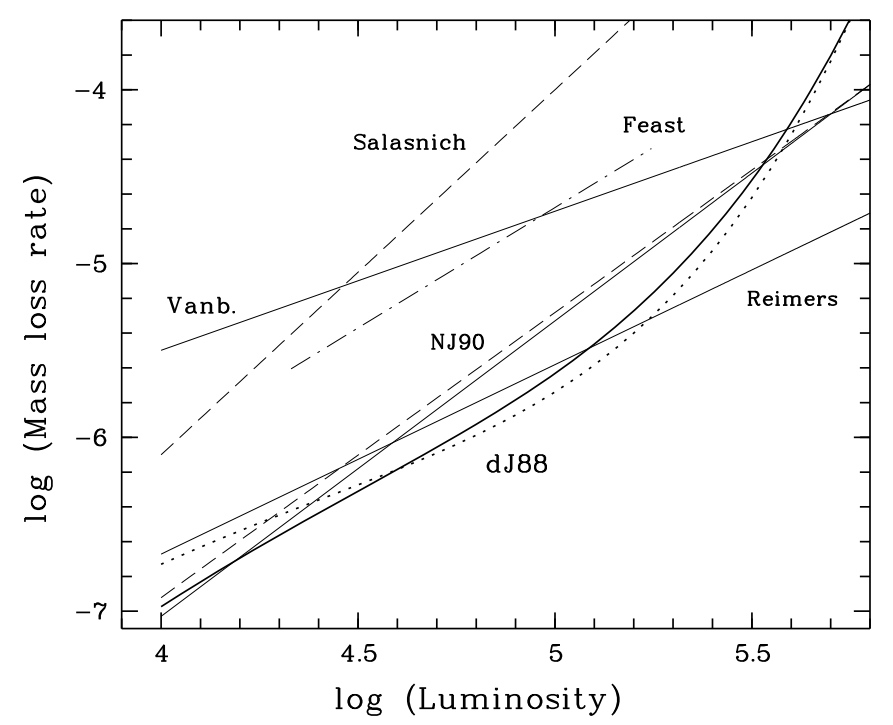

Fig. 1. Comparison of several prescriptions for the RSG mass-loss rates (unit $M_{\odot} \mathrm{yr}^{-1}$ ) plotted against luminosity (in $L_{\odot}$ ). The de Jager prescription is represented by the two curved lines (solid line $T_{\text {eff }}=4000 \mathrm{~K}$, dotted line $T_{\text {eff }}=3500 \mathrm{~K}$ ).

on stellar mass, luminosity, and temperature is more explicit. Nieuwenhuijzen \& de Jager (1990) find:

$\log \dot{M}=-7.93+1.64 \log L+0.16 \log M-1.61 \log T_{\text {eff }}$.

It can be seen in the above formula that $\dot{M}$ depends very little on $M$, because for $M=9 M_{\odot}$ and $25 M_{\odot}$, the second term is equal to 0.15 and 0.22 respectively. This represents a difference of only 17 percent on $\dot{M}$, and consequently this mass term can be considered to be constant and equal to 0.18 . Then, a more explicit formulation is

$\dot{M}=5.6 \times 10^{-6}\left(L / 10^{5}\right)^{1.64}\left(T_{\mathrm{eff}} / 3500\right)^{-1.61}$.

The rate of NJ90 is plotted in Fig. 1 for $T_{\text {eff }}=3750 \mathrm{~K}$. For other temperatures, this rate does not change much: for effective temperatures of 3500 or $4000 \mathrm{~K}$, the NJ90 line in Fig. 1 would be shifted up or down by only 0.05 in the $y$-axis. This NJ90 rate is not very different from the de Jager rate: the largest difference is about a factor of 2 at $L \sim 10^{5}$. Also Nieuwenhuijzen \& de Jager note that for cool evolved stars the dJ88 rate is more accurate than the NJ90 one in fitting the $\dot{M}$ observational measurements (their Table 2).

We also plotted in Fig. 1 the rate adopted in the recent Geneva models (Hirschi, private communication). This rate is represented by a continuous straight line located very close to, and just below the NJ90 line. This Geneva rate is practically identical to the NJ90 one, but there is no dependency on $T_{\text {eff }}$. The Geneva rate is $\dot{M}=4.7 \times 10^{-6}\left(L / 10^{5}\right)^{1.7}$.

Figure 1 also shows the Reimers law (Reimers 1975; Kudritzki \& Reimers 1978)

$\dot{M}=5.5 \times 10^{-13} L R / M$,

with $L, R$, and $M$ in solar units. Because we restrict our survey to RSGs, this relation can be simplified: $R$ is equal to $L^{0.5} \times\left(T_{\text {eff }} / 5770\right)^{-2}$ and the factor depending on $T_{\text {eff }}$ does not vary much $\left(2.72\right.$ and 2.08 for $T_{\text {eff }}=3500$ and $4000 \mathrm{~K}$ respectively). In addition, for the RSGs studied here, there is an approximate relation between mass and luminosity. For $M=9 M_{\odot}$, $L \approx 2.5 \times 10^{4} L_{\odot}$, and for $M=25 M_{\odot}, L \approx 3 \times 10^{5} L_{\odot}$ (see, for 
example, Fig. 8 of Massey et al. 2009, lower panels). We find $M \approx 0.14 L^{0.41}$. The Reimers relation for an average temperature $T_{\text {eff }}=3750 \mathrm{~K}$ is

$\dot{M}=2.6 \times 10^{-6}\left(L / 10^{5}\right)^{1.09}$.

This relation is plotted in Fig. 1, and obviously the Reimers rate is close to the dJ88 rate (within a factor of 2 for $L \leq 2 \times 10^{5} L_{\odot}$ ), but the latter is significantly stronger for higher luminosities. The Reimers lines corresponding to other temperatures would be 0.060 (in $\log$ ) above and 0.056 below the plotted line, for 3500 and $4000 \mathrm{~K}$ respectively. This shows that the dependence on temperature is quite small compared to the observational uncertainty on measured mass-loss rates considered below (typically a factor 2 or 3 ).

There exist other mass-loss prescriptions. Feast (1992) noted that there is a very good correlation between the pulsation period $P$ of 15 RSGs of the Large Magellanic Cloud and their luminosities $L$ based on $J H K$ and IRAS data and estimated by Reid et al. (1990). The mass-loss rates published by Reid et al. for these stars also correlate fairly well with $P$. A relation linking $\dot{M}$ and $L$ is derivable and given in Eq. (12) of Salasnich et al. (1999). This relation (plotted in Fig. 1) can be written as

$\dot{M}=2.1 \times 10^{-5}\left(L / 10^{5}\right)^{1.385}$.

A prescription based also on the LMC RSGs and the work of Reid et al. (1990) is used by Vanbeveren et al. (1998; see also Vanbeveren et al. 2007). It is labelled "Vanb." in Fig. 1. This rate agrees with the Feast rate for $L \sim 10^{5} L_{\odot}$, but its slope is lower. Its expression is

$$
\dot{M}=2.0 \times 10^{-5}\left(L / 10^{5}\right)^{0.80} \text {. }
$$

Another mass-loss prescription has been proposed by Salasnich et al. (1999), who adopt the Feast relation, but take a gas-todust mass ratio that increases with luminosity. Typically, this ratio would be 200 for AGB stars, but could increase to 1000 for RSGs with a luminosity of $10^{5} L_{\odot}$. Consequently, the Salasnich prescription implies very high mass-loss rates. For $L \sim 10^{5} L_{\odot}$, this prescription gives $10^{-4} M_{\odot} \mathrm{yr}^{-1}$, about a factor of 50 above the de Jager rate. The Salasnich law is

$\dot{M}=1.00 \times 10^{-4}\left(L / 10^{5}\right)^{2.1}$.

Finally, van Loon et al. (2005) derived a mass-loss law for AGB stars and RSGs, but it cannot be applied if the circumstellar envelope is not very dusty. Nevertheless, the rate of van Loon et al. (2005) is interesting to compare with the de Jager rate, and this will be done with more details in the discussion. In the next section, we compare the de Jager rate with modern mass-loss measurements.

\section{Comparison of the de Jager rate with observations}

\subsection{The 60- $\mu \mathrm{m}$ excess emission of RSGs in the solar neighbourhood}

The RSGs of the solar neighbourhood are key targets for investigating mass loss, either because they are close to us like Betelgeuse or Antares, or because the membership to an OB association gives a distance indication and confirms the object to be a massive star. These RSGs have been originally listed by Humphreys (1978). Jura \& Kleinman (1990) investigated mass loss of the most luminous RSGs. They consider a list of $21 \mathrm{ob}-$ jects more luminous than $10^{5} L_{\odot}$ and estimate mass loss with the IRAS $60 \mu \mathrm{m}$ flux. Josselin et al. (2000) consider 65 RSGs, present photometric $J H K L$ measurements of most of them, and detect the $\mathrm{CO}$ millimeter emission in several cases. One of their conclusions is a large scatter of $\dot{M}$ for a given luminosity, a property that was also noted by JK90. Levesque et al. (2005) have revised effective temperatures, distances, and interstellar extinction for 74 RSGs.

A first list of RSGs was built from the list of 74 objects of LM05, supplemented with 21 objects from JB00. For these 95 sources, we collected optical (UBVI) and near-infrared photometry $(J H K L)$, preferentially from Lee (1970). Data from Simbad, 2MASS, JB00 and Gezari et al. (1993) were also used. At midinfrared wavelengths, IRAS measurements were adopted. Then, for most of the stars, photometry from the $U$ band to the $60 \mu \mathrm{m}$ band is available. Almost all these stars belong to OB associations, and the distance moduli and interstellar absorption given by LM05 were adopted. When a star was not examined in the work of LM05, we took the distance modulus of the association from Humphreys (1978) and the reddening was derived from the averaged reddening of the OB-type stars of the association. For several objects (e.g., VY CMa, VX Sgr), the distance and absorption come from recent, individual determinations.

From this primary list of 95 objects, we are interested in those presenting evidence of mass loss. For that purpose, we selected objects presenting an infrared excess, measured by the ratio of $12 \mu \mathrm{m}$ flux to the dereddened $K$-band flux. Following JK90, only stars with $f_{12} / f_{K}$ larger than 0.1 were selected. In addition, we imposed $f_{60} \geq 2 \mathrm{Jy}$, in order to work with reliable $60 \mu \mathrm{m}$ fluxes. This resulted in a list of 39 objects for which the photometric data are given in Appendix B. The characteristics of these 39 objects are given in Table 1. An additional remarkable RSG, NML Cyg, will be examined in the next subsection.

In order to compare the mass-loss rate of these RSGs with the de Jager prescription, we estimate $\dot{M}$ from the infrared excess at $60 \mu \mathrm{m}$ with the formulation of Jura and collaborators (JK90):

$\dot{M}_{60 \mu \mathrm{m}}=1.7 \times 10^{-7} V_{15} D_{\mathrm{kpc}}^{2} f_{60}\left(\lambda_{10} / L_{4}\right)^{1 / 2}$,

where $\dot{M}_{60 \mu \mathrm{m}}$ is in $M_{\odot} \mathrm{yr}^{-1}, V_{15}$ is the wind expansion velocity normalized to $15 \mathrm{~km} \mathrm{~s}^{-1}, D_{\mathrm{kpc}}$ is the distance in $\mathrm{kpc}, f_{60}$ is the IRAS flux at $60 \mu \mathrm{m}$ in Jy (assumed to dominate the photospheric flux at this wavelength), $\lambda_{10}$ is equal to the mean wavelength $\lambda_{\mathrm{m}}$ of the object spectral energy distribution, normalized to $10 \mu \mathrm{m}$, and $L_{4}$ is the luminosity in units of $10^{4} L_{\odot}$. This formula is valid for a gas-to-dust mass ratio of 200 , which corresponds to complete condensation of refractory elements for solar abundances, and we adopt this ratio here.

The wind speed in Jura's formula is known for only a few RSGs (see Table 2). For all others, we choose to scale the value for this speed with the luminosity, and we adopt $V \propto L^{0.35}$ following predictions of dust-driven wind modelling (Habing et al. 1994). For the RSGs of the solar neighbourhood, we find that $V \simeq 20 \times\left(L / 10^{5}\right)^{0.35} \mathrm{~km} \mathrm{~s}^{-1}$ roughly represents the observed values. For the LMC RSGs considered below, we find $V \simeq 14 \times\left(L / 10^{5}\right)^{0.35} \mathrm{~km} \mathrm{~s}^{-1}$ (see Appendix C for details).

Concerning the luminosity and mean wavelength, these quantities are calculated by integrating over the spectral energy distribution. We use the UBVIJHKL magnitudes and IRAS fluxes and correct the magnitudes for interstellar reddening following the extinction law of Cardelli et al. (1989). Between the central wavelengths of these spectral bands, interpolation is done linearly on the relation of $\log \left(\lambda f_{\lambda}\right)$ as a function of $\log (\lambda)$. The accuracy of the derived luminosities (listed as $L_{\text {phot }}$ 
Table 1. Properties of the RSGs.

\begin{tabular}{|c|c|c|c|c|c|c|c|c|c|c|}
\hline Name & $\begin{array}{r}m-M \\
(\mathrm{mag})\end{array}$ & $\begin{array}{r}E_{B-V} \\
(\mathrm{mag})\end{array}$ & $f_{12} / f_{\mathrm{K}}$ & $\begin{array}{c}V \\
\left(\mathrm{~km} \mathrm{~s}^{-1}\right)\end{array}$ & $\begin{array}{r}D \\
(\mathrm{kpc})\end{array}$ & $\begin{array}{r}f_{60} \\
(\mathrm{Jy})\end{array}$ & $\begin{array}{r}\lambda_{m} \\
(\mu \mathrm{m})\end{array}$ & $\begin{array}{l}L_{\text {Lev }} \\
\left(L_{\odot}\right)\end{array}$ & $\begin{array}{l}L_{\text {phot }} \\
\left(L_{\odot}\right)\end{array}$ & $\begin{array}{r}\dot{M} \\
\left(M_{\odot} \mathrm{yr}^{-1}\right)\end{array}$ \\
\hline V589 Cas & 11.50 & 0.78 & 0.156 & 14 & 2.00 & 3.61 & 1.72 & 52000 & 35000 & $5.0 \times 10^{-7}$ \\
\hline BU Per & 11.40 & 0.66 & 0.435 & 14 & 1.90 & 5.23 & 2.20 & 58000 & 38000 & $7.4 \times 10^{-7}$ \\
\hline SU Per & 11.40 & 0.66 & 0.241 & 19 & 1.90 & 6.87 & 1.77 & 90000 & 85000 & $7.7 \times 10^{-7}$ \\
\hline RS Per & 11.90 & 0.56 & 0.417 & 20 & 2.40 & 9.93 & 2.28 & 144000 & 95000 & $2.0 \times 10^{-6}$ \\
\hline S Per & 11.39 & 0.66 & 1.226 & 20 & 1.90 & 40.59 & 3.67 & 81000 & 86000 & $6.8 \times 10^{-6}$ \\
\hline V441 Per & 11.40 & 0.66 & 0.156 & 16 & 1.90 & 3.54 & 1.69 & 66000 & 50000 & $4.2 \times 10^{-7}$ \\
\hline YZ Per & 11.40 & 0.66 & 0.291 & 16 & 1.90 & 5.28 & 1.86 & 48000 & 55000 & $6.5 \times 10^{-7}$ \\
\hline W Per & 11.40 & 0.66 & 0.495 & 16 & 1.90 & 14.87 & 2.55 & 54000 & 56000 & $2.1 \times 10^{-6}$ \\
\hline $\mathrm{BD}+57647$ & 11.40 & 0.66 & 0.351 & 14 & 1.90 & 6.47 & 2.24 & 80000 & 37000 & $9.3 \times 10^{-7}$ \\
\hline NO Aur & 10.70 & 0.47 & 0.146 & 18 & 1.38 & 5.12 & 1.62 & 67000 & 73000 & $2.9 \times 10^{-7}$ \\
\hline$\alpha$ Ori & 5.57 & 0.18 & 0.177 & 15 & 0.130 & 299.00 & 1.64 & $\ldots$ & 56000 & $1.5 \times 10^{-7}$ \\
\hline TV Gem & 10.70 & 0.66 & 0.294 & 19 & 1.38 & 6.06 & 1.69 & 100000 & 84000 & $3.5 \times 10^{-7}$ \\
\hline BU Gem & 10.70 & 0.66 & 0.248 & 19 & 1.38 & 10.50 & 1.62 & 83000 & 86000 & $5.9 \times 10^{-7}$ \\
\hline V384 Pup & 13.00 & 0.57 & 0.260 & 18 & 4.00 & 2.76 & 1.84 & 37000 & 75000 & $1.4 \times 10^{-6}$ \\
\hline CK Car & 11.70 & 0.55 & 0.531 & 22 & 2.20 & 13.98 & 2.11 & 161000 & 123000 & $2.1 \times 10^{-6}$ \\
\hline V602 Car & 11.60 & 0.48 & 0.596 & 22 & 2.10 & 12.40 & 2.61 & 105000 & 124000 & $1.9 \times 10^{-6}$ \\
\hline V396 Cen & 11.60 & 0.75 & 0.201 & 23 & 2.10 & 4.98 & 1.73 & 164000 & 140000 & $6.2 \times 10^{-7}$ \\
\hline KW Sgr & 12.40 & 0.92 & 1.218 & 27 & 3.00 & 18.39 & 2.81 & 363000 & 228000 & $5.6 \times 10^{-6}$ \\
\hline NR Vul & 11.80 & 0.94 & 0.590 & 21 & 2.30 & 12.28 & 2.26 & 224000 & 111000 & $2.2 \times 10^{-6}$ \\
\hline BI Cyg & 11.00 & 0.93 & 0.671 & 22 & 1.58 & 51.23 & 2.67 & 226000 & 123000 & $4.6 \times 10^{-6}$ \\
\hline KY Cyg & 11.00 & 0.93 & 0.702 & 22 & 1.58 & 50.74 & 3.15 & 272000 & 138000 & $4.9 \times 10^{-6}$ \\
\hline RW Cyg & 10.60 & 1.22 & 0.481 & 23 & 1.32 & 60.69 & 1.96 & 144000 & 145000 & $3.2 \times 10^{-6}$ \\
\hline$\mu$ Cep & 9.70 & 0.69 & 0.361 & 20 & 0.87 & 127.00 & 1.69 & 340000 & 410000 & $1.4 \times 10^{-6}$ \\
\hline V354 Cep & 12.20 & 0.63 & 0.566 & 18 & 2.75 & 8.01 & 2.89 & 369000 & 76000 & $2.4 \times 10^{-6}$ \\
\hline V355 Cep & 12.20 & 0.63 & 0.292 & 14 & 2.75 & 3.27 & 2.38 & 94000 & 37000 & $1.0 \times 10^{-6}$ \\
\hline $\mathrm{PZCas}$ & 11.90 & 0.69 & 1.217 & 30 & 2.40 & 96.48 & 3.57 & 212000 & 193000 & $2.6 \times 10^{-5}$ \\
\hline TZ Cas & 11.90 & 0.69 & 0.541 & 19 & 2.40 & 9.47 & 2.38 & 98000 & 83000 & $2.0 \times 10^{-6}$ \\
\hline EV Car & 13.13 & 0.51 & 0.745 & 39 & 4.20 & 25.87 & 2.57 & $\ldots$ & 675000 & $1.3 \times 10^{-5}$ \\
\hline HS Cas & 12.00 & 0.83 & 0.275 & 17 & 2.50 & 3.51 & 1.92 & $\ldots$ & 59000 & $7.5 \times 10^{-7}$ \\
\hline XX Per & 11.14 & 0.66 & 0.492 & 16 & 1.69 & 4.23 & 2.03 & $\ldots$ & 50000 & $4.3 \times 10^{-7}$ \\
\hline KK Per & 11.14 & 0.66 & 0.117 & 16 & 1.69 & 2.23 & 1.59 & $\ldots$ & 50000 & $2.0 \times 10^{-7}$ \\
\hline AD Per & 11.14 & 0.66 & 0.164 & 15 & 1.69 & 2.85 & 1.66 & $\ldots$ & 42000 & $2.7 \times 10^{-7}$ \\
\hline PR Per & 11.14 & 0.66 & 0.131 & 14 & 1.69 & 2.37 & 1.55 & $\begin{array}{l}\cdots \\
\cdots\end{array}$ & 34000 & $2.3 \times 10^{-7}$ \\
\hline GP Cas & 11.40 & 0.66 & 0.200 & 15 & 1.90 & 4.45 & 2.01 & $\ldots$ & 43000 & $5.9 \times 10^{-7}$ \\
\hline VY CMa & 10.28 & 0.47 & 6.959 & 47 & 1.14 & 1453.00 & 7.77 & $\ldots$ & 295000 & $1.6 \times 10^{-4}$ \\
\hline$\alpha \mathrm{Sco}$ & 6.34 & 0.10 & 0.165 & 17 & 0.185 & 115.50 & 1.73 & $\begin{array}{l}\cdots \\
\cdots\end{array}$ & 71000 & $1.2 \times 10^{-7}$ \\
\hline VX Sgr & 10.98 & 0.52 & 2.334 & 25 & 1.57 & 262.70 & 4.14 & $\begin{array}{l}\cdots \\
\cdots\end{array}$ & 343000 & $2.0 \times 10^{-5}$ \\
\hline Case 49 & 11.70 & 0.87 & 0.155 & 15 & 2.19 & 6.58 & 2.01 & $\begin{array}{l}\cdots \\
\cdots\end{array}$ & 42000 & $1.1 \times 10^{-6}$ \\
\hline U Lac & 12.20 & 0.63 & 0.685 & 23 & 2.75 & 9.04 & 2.51 & $\begin{array}{l}\cdots \\
\cdots\end{array}$ & 147000 & $2.3 \times 10^{-6}$ \\
\hline
\end{tabular}

Notes. The first 27 stars are from LM05, while the following 12 stars are from JB00. The column marked $V$ is the assumed wind speed. The column marked $\lambda_{m}$ is the flux averaged wavelength. $L_{\mathrm{Lev}}$ is the luminosity given in LM05 (from their $M_{\mathrm{bol}}$ ) and $L_{\mathrm{phot}}$ is the luminosity obtained by integrating the $U B V I J H K L+$ IRAS photometry. $\dot{M}$ is derived with Jura's formula, where the used luminosity is $L_{\text {phot }}$.

in Table 1) depends on several factors. Once the distance is fixed with the distance modulus, factors like photometric uncertainties, variability or errors on $E_{B-V}$ introduce uncertainty. For example, adopting $J H K$ magnitudes smaller by 0.2 mag results a luminosity higher by $\lesssim 11 \%$, and a decrease of $\dot{M}$ by $\$ 7 \%$. Similarly, increasing $E_{B-V}$ by 0.25 mag implies an increase in $L$ by $\lesssim 40 \%$, and a decrease in $\dot{M}$ by $\lesssim 16 \%$. These variations are smaller than the accuracy of Jura's formula.

In Table 1 we included the luminosities from LM05 (column $L_{\text {Lev }}$ for the 26 objects originating from their study). Many determinations of luminosity agree very well between their and our studies. However, there are also a few significant discrepancies, up to a factor of 5 for V354 Cep. Because we use the same distance and reddening and almost the same photometry, the discrepancy must originate from the method for deriving $L$, but we could not trace exactly where the differences come from.
Consequently, we used our luminosities for plotting $\dot{M}$ versus $L$ (Fig. 2).

It can be seen in Fig. 2 that if we omit four exceptions labelled 1, 2, 3, and 7, the majority of points are clustered near the de Jager prescription line. For $\log L<5.2$, the de Jager line slightly overestimates the mass-loss rates (by a factor $\sim 1.5$ ), while for $\log L>5.2$, the de Jager line passes below the stars PZ Cas and VY CMa, but above KW Sgr and VX Sgr, and decreasing the dJ88 rate by 1.5 would not improve the situation.

The stars $\alpha$ Ori, $\alpha$ Sco, and $\mu$ Cep (labelled 1, 2, and 3 respectively) present a low $\dot{M}$ here, but observations of their circumstellar gas suggest that they are plausibly deficient in grains (see next section). We note that our estimate of $\dot{M}$ for $\mu$ Cep, based on the $60 \mu \mathrm{m}$ flux, agrees well with that of de Wit et al. (2008) derived from imaging at $24 \mu \mathrm{m}$, after appropriate scaling of the outflow velocity. 
N. Mauron and E. Josselin: The mass-loss rates of red supergiants

Table 2. Characteristics of RSGs for which a mass-loss rate has been measured by observing the circumstellar gas.

\begin{tabular}{|c|c|c|c|c|c|c|c|c|c|c|c|c|}
\hline \multirow{2}{*}{$\begin{array}{l}\text { Name } \\
\text { (1) }\end{array}$} & \multirow{2}{*}{$\begin{array}{l}D \\
{[\mathrm{kpc}]} \\
(2)\end{array}$} & \multirow{2}{*}{$\begin{array}{l}E_{B-V} \\
{[\mathrm{mag}]} \\
(3)\end{array}$} & \multirow{2}{*}{$\begin{array}{c}V \\
{\left[\mathrm{~km} \mathrm{~s}^{-1}\right]} \\
(4)\end{array}$} & \multirow{2}{*}{$\begin{array}{r}F_{60} \\
\mathrm{Jy} \\
(5)\end{array}$} & \multirow{2}{*}{$\begin{array}{c}\lambda_{\mathrm{m}} \\
{[\mu \mathrm{m}]} \\
(6)\end{array}$} & \multirow{2}{*}{$\begin{array}{r}L_{\text {phot }} \\
{\left[L_{\odot}\right]} \\
(7)\end{array}$} & \multirow{2}{*}{$\begin{array}{l}\dot{M}_{60 \mu \mathrm{m}} \\
{\left[10^{-6} M_{\odot}\right.} \\
\quad(8)\end{array}$} & \multirow{2}{*}{$\begin{array}{r}\dot{M}_{\text {gas }} \\
\left.\mathrm{yr}^{-1}\right] \\
(9)\end{array}$} & \multirow{2}{*}{$\begin{array}{l}\text { Method } \\
\text { (10) }\end{array}$} & \multicolumn{3}{|c|}{ References } \\
\hline & & & & & & & & & & (11) & (12) & (13) \\
\hline$\alpha$ Ori & 0.130 & 18 & 15 & 299 & 1.6 & 56000 & 0.15 & 1.5 & $\mathrm{HI}$ C I & 1 & 2 & 3 \\
\hline$\alpha$ Sco & 185 & .10 & 17 & 115 & 1.7 & 71000 & 0.12 & 1.0 & H II reg. & 1 & 4 & 5 \\
\hline S Per & 1.90 & 0.66 & 20 & 41 & 3.67 & 86000 & 6.8 & 7.5 & $\mathrm{CO}$ & 6 & 6 & 7 \\
\hline PZ Cas & 2.40 & 0.69 & 30 & 96 & 3.57 & 193000 & 26 & 8.3 & $\mathrm{CO}$ & 8 & 8 & 7 \\
\hline VY CMa & 1.14 & 0.47 & 47 & 1453 & 7.77 & 295000 & 160 & 40 & $\mathrm{CO}$ & 9 & 10 & 11 \\
\hline NML Cyg & 1.74 & 1.20 & 34 & 1030 & 9.80 & 320000 & 210 & 140 & $\mathrm{CO}$ & 12 & 13 & 14 \\
\hline VX Sgr & 1.57 & 0.52 & 25 & 263 & 4.14 & 340000 & 20 & 11 & $\mathrm{CO}$ & 15 & 16 & 17 \\
\hline$\mu$ Сер & 0.87 & 0.69 & 20 & 127 & 1.69 & 410000 & 1.4 & 5.0 & K I Na I & 6 & 6 & 18 \\
\hline
\end{tabular}

Notes. Column $(11)=$ reference for distance; Col. $(12)=$ reference for $E_{B-V}$; Col. $(13)=$ reference for mass-loss rate $\dot{M}_{\text {gas }}$. The IRAS data of NML Cyg were taken from Schuster et al. (2009).

References. (1) Hipparcos parallax; (2) Lambert et al. (1984); (3) Huggins et al. (1994); (4) assumed; (5) Reimers et al. (2008); (6) LM05; (7) Woodhams (1993); (8) JB00; (9) Choi et al. (2008); (10) assuming $E_{B-V}=0.47$ per kpc; (11) Decin et al. (2006) and see text; (12) Massey \& Thomson (1991); (13) Blöcker et al. (2001); (14) Knapp et al. (1982); (15) Chen et al. (2007); (16) Humphreys (1978); (17) Knapp et al. (1989); (18) Guilain \& Mauron (1996).

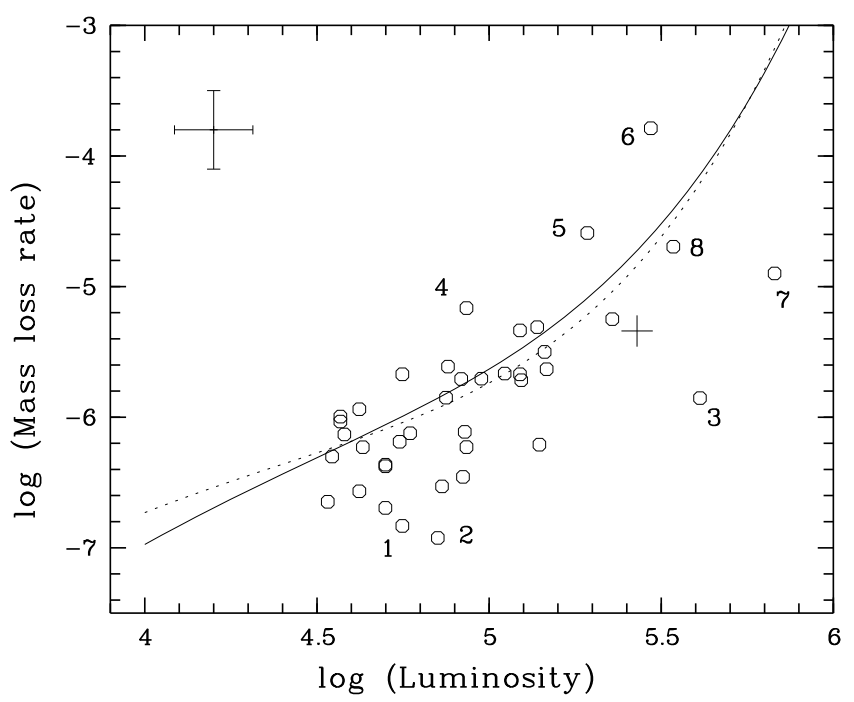

Fig. 2. Comparison of de Jager rate (plotted for $T_{\text {eff }}=3500 \mathrm{~K}$ and $4000 \mathrm{~K})$ with mass-loss rates of 39 RSGs listed in Table 1. The mass-loss rates are based on the study of dust and obtained with Jura's formula. The labels indicate a few wellknown RSGs as follows: 1: $\alpha$ Ori; 2: $\alpha$ Sco, 3: $\mu$ Cep, 4: S Per, 5: PZ Cas, 6: VY CMa, 7: EV Car (at $4.2 \mathrm{kpc}$ ); 8: VX Sgr. The small cross represents EV Car if a smaller distance of $2.5 \mathrm{kpc}$ is adopted. At the upper left corner, error bars represent uncertainties of \pm 30 percent in luminosity, and a factor of \pm 2 in $\dot{M}$.

There is also one star at very high luminosity: EV Car (labelled 7), with $L=6.7 \times 10^{5} L_{\odot}$ and $\dot{M}=1.3 \times 10^{-5} M_{\odot} \mathrm{yr}^{-1}$. The huge luminosity is owing to the distance modulus given by Humphreys (1978), $(m-M)=13.3$, corresponding to $4.2 \mathrm{kpc}$. This puts the star far beyond the Car OB1 association. If we had taken the distance of this association $(2.5 \mathrm{kpc}$, adopted by JK90), we would have found $L=2.4 \times 10^{5} L_{\odot}$ and $\dot{M}=4.6 \times 10^{-6} M_{\odot} \mathrm{yr}^{-1}$, and the location of the star in Fig. 2 would be very close to the de Jager line. But to our knowledge, there is no observational evidence for EV Car to be member of Car OB1.

\subsection{Circumstellar gas of RSGs in the solar neighbourhood}

Only a few RSG winds have been detected through observation of the circumstellar gas. Methods of detection include measurements of the $\mathrm{HI} 21 \mathrm{~cm}$ emission (details on observations and mass-loss determination can be found in Bowers \& Knapp 1987), millimetric emission of CO (Olofsson 2004; Ramstedt et al. 2008), submillimeter emission of C I (Keene et al. 1993; Huggins et al. 1994), emission of scattered resonance lines of Na I and KI (Mauron 1990; Guilain \& Mauron 1996; Gustafsson et al. 1997), and observation of the H II region around Antares (Kudritzki \& Reimers 1978; Reimers et al. 2008).

For eight stars we collected in Table 2 the mass-loss rates obtained with these methods, giving detailed reference for the adopted parameters ( $D$ and $E_{B-V}$ ) used to derive luminosities. When $\dot{M}$ was obtained in original papers with a different distance, we assumed $\dot{M} \propto D^{2}$. The expansion velocities $V$ are from Huggins et al. (1994) for $\alpha$ Ori, Woodhams (1993) for S Per and PZ Cas, and from Kemper et al. (2003) for VY CMa, VX Sgr, NML Cyg. Finally, for $\mu$ Cep, the situation is unclear (for details see Bernat 1981; the discussion in Le Borgne \& Mauron 1989; and Kemper et al. 2003) and we adopted $20 \mathrm{~km} \mathrm{~s}^{-1}$.

Several remarks have to be made on individual objects. For the most observed star ( $\alpha$ Ori), there is good agreement among the rates based on HI, CI and KI (Bowers \& Knapp 1987; Huggins et al. 1994; Glassgold \& Huggins 1986; Plez $\&$ Lambert 2002). When the distance given by Hipparcos is adopted (parallax $7.63 \pm 1.64$ mas, giving $130 \mathrm{pc} \pm 30 \mathrm{pc}$ ), one obtains $\dot{M} \approx 1$ to $2 \times 10^{-6} M_{\odot} \mathrm{yr}^{-1}$. The distance proposed by Harper et al. (2008) is $197 \pm 45$ pc and would imply $\dot{M} \sim$ $3 \times 10^{-6} M_{\odot} \mathrm{yr}^{-1}$. For this star, the interstellar extinction $A_{\mathrm{V}}$ is not very well determined, being between 0.3 and 0.8 mag according to Lambert et al. (1984). We have chosen the average of these values implying $L=56000 L_{\odot}$, but the main uncertainty comes from the error on parallax, and $L$ is known within \pm 50 percent.

In the case of VX Sgr, the distance has recently been measured by Chen et al. (2007) with $\mathrm{SiO}$ maser proper motions $(D=1.57 \pm 0.27 \mathrm{kpc})$, and this distance means that the star belongs to the Sgr OB1 association. The average interstellar extinction to the OB stars of this association is $E_{B-V}=0.5$ (from data of Humphreys 1978), and the luminosity is $3.4 \times 10^{5} L_{\odot}$. 


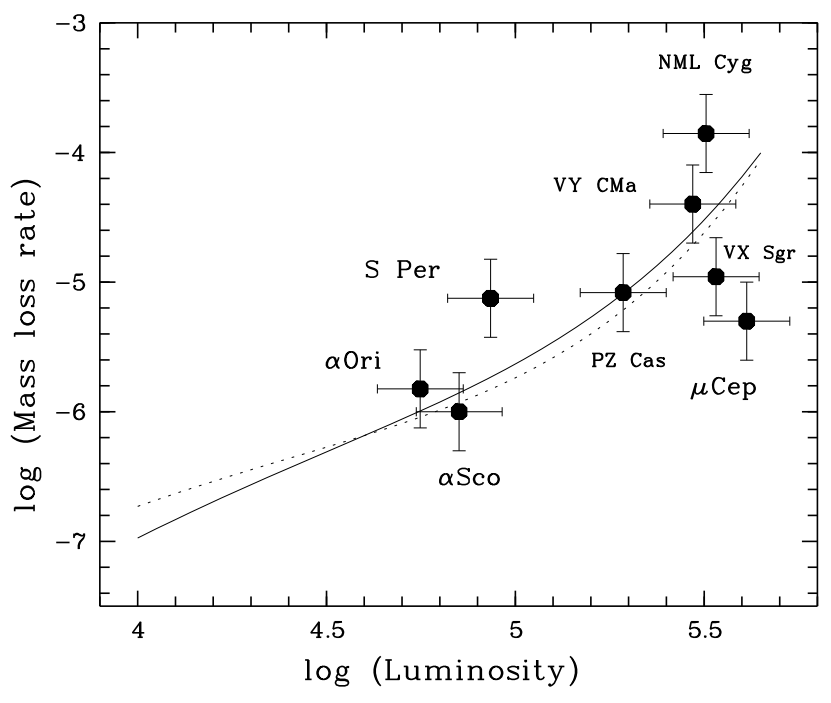

Fig. 3. Mass-loss rates (from observations of the gas) versus luminosity for the eight RSGs of Table 2. The lines are the de Jager rates for $4000 \mathrm{~K}$ (solid line) and $3500 \mathrm{~K}$ (dotted line).

For VY CMa, a distance of $1.14 \pm 0.10 \mathrm{kpc}$ has been obtained by Choi et al. (2008) through astrometric monitoring of $\mathrm{H}_{2} \mathrm{O}$ masers. This distance is smaller than the one often used. For example, Decin et al. (2006) have adopted $1.50 \mathrm{kpc}$ for their detailed study of circumstellar CO lines. The ejection rate obtained by Decin et al. is highly variable. The episode with the highest mass-loss rate $\left(\sim 3 \times 10^{-4} M_{\odot} \mathrm{yr}^{-1}\right)$ has a duration of only $100 \mathrm{yr}$. The average mass-loss rate is about $7 \times 10^{-5} M_{\odot} \mathrm{yr}^{-1}$. Scaled to the distance of $1.14 \mathrm{kpc}$, the average rate becomes $4 \times 10^{-5} M_{\odot} \mathrm{yr}^{-1}$. The new smaller distance means that the star is less luminous than previously believed. We find $L=2.9 \times 10^{5} L_{\odot}$. Because the star is extremely red (the flux averaged wavelength is around $8 \mu \mathrm{m}$, compared to $\sim 2 \mu \mathrm{m}$ for the majority of objects), this luminosity does not depend much on the choice of the extinction: for $E_{B-V}$ in the range 0 to 1 , the luminosity is between 2.7 and $3.5 \times 10^{5} L_{\odot}$.

The points representing these eight stars with measured gas mass-loss rates as a function of luminosity are shown in Fig. 3. The error bars correspond to \pm 30 percent in luminosity and a factor of \pm 2 of uncertainty on the mass-loss rate. The actual uncertainties on $\dot{M}$ are probably higher than that but difficult to quantify. The points are not very far from the de Jager line. Betelgeuse and Antares agree for $\dot{M} \approx 10^{-6} M_{\odot} \mathrm{yr}^{-1}$ at a luminosity of $\sim 65000 L_{\odot}$. S Per is a factor of 4 above the line and $\mu \mathrm{Cep}$ is a factor of 10 below the line. It can be seen that on average the de Jager prescription agrees reasonably well with measurements of the gas mass-loss rates.

Because $\dot{M}_{60} \mu \mathrm{m}$ was used in the previous section, we can compare it for these stars with the mass-loss rates derived from observation of the gas $\dot{M}_{\text {gas }}$. This is done in Fig. 4 and Table 2. Figure 4 shows that the agreement between the two rates is satisfactory for some stars (S Per, NML Cyg, VX Sgr), but there are also differences of a factor of $\sim 4$ for VY CMa and $\mu$ Cep. These differences may arise because different regions of the envelope are probed when comparing different techniques. For $\alpha$ Ori and $\alpha$ Sco, $\dot{M}_{60} \mu \mathrm{m}$ is too low by a factor of $\sim 10$, plausibly due to incomplete dust formation. There is a trend in Fig. 4 that for low $\dot{M}$, grain condensation may be incomplete, but obviously many additional sources should be examined with independent estimates of the dust mass-loss rate and the gas mass-loss rate to confirm this trend.

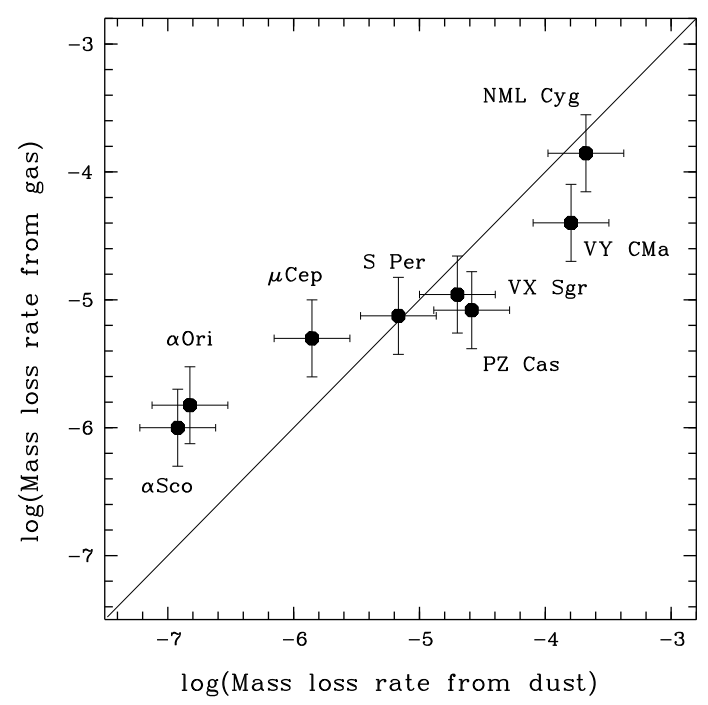

Fig. 4. Mass-loss rates from observation of the gas versus mass-loss rate from observation of the dust (the $60 \mu \mathrm{m}$ emission) for the eight RSGs of Table 2.

\subsection{Infrared measurements in the Large Magellanic Cloud}

The mass-loss rate of RSGs in the Large Magellanic Cloud (LMC) has been investigated in several works, with various data and various methods. All works are based on the circumstellar dust emission. Reid et al. (1990) used IRAS fluxes and Jura's formula. van Loon et al. (2005) exploited MSX, ISO and IRAS fluxes (their Table 3) and analysed the spectral energy distribution with the dust radiative transfer code DUSTY, which provides $\dot{M}$. More recently, Groenewegen et al. (2009) exploited Spitzer results in addition to all previously obtained data. In particular, Spitzer data include photometry from 3.6 to $24 \mu \mathrm{m}$ and spectroscopy from 5.2 to $37 \mu \mathrm{m}$. Groenewegen et al. (2009) fit the data also with dust radiative transfer models. (The work of Bonanos et al. (2010) also treats mass loss of RSGs in the Magellanic Clouds and is considered in the Discussion.)

The mass-loss rates given in the works cited above cannot be compared directly, because their assumed parameters, such as the expansion velocity of the wind or the gas-to-dust mass ratio are different. We assume here that the LMC RSGs have a metallicity lower than that of the Sun so that complete condensation of refractory metals leads to a gas-to-dust mass ratio $\psi=500$. We also assume that all RSGs have a wind velocity $v(L)$ depending on the luminosity $L$ as found in Appendix C i.e. $v(L)=14\left(L / 10^{5}\right)^{0.35} \mathrm{~km} \mathrm{~s}^{-1}$. Consequently, we multiplied the $\dot{M}$ of Reid et al. (1990) by $2.5 \times v(L) / 15$ (because Jura's formula used by Reid et al. supposes $\psi=200$ and a wind speed of $15 \mathrm{~km} \mathrm{~s}^{-1}$ ). We multiplied the $\dot{M}$ of van Loon et al. (2005) by $v(L) / v^{\prime}$ where $v^{\prime}$ is their published wind velocity provided by DUSTY. Finally, we multiplied by $2.5 \times v(L) / 10$ the $\dot{M}$ of Groenewegen et al. (2009) because they adopt $\psi=200$ and a wind speed of $10 \mathrm{~km} \mathrm{~s}^{-1}$.

The results are given in Tables 3-5. As far as possible, we did not include AGB stars in these tables. More precisely, we followed Groenewegen et al. (2009) by considering that all objects with $L \gtrsim 1.2 \times 10^{5} L_{\odot}$ are genuine RSGs, and that objects with $L$ between $\sim 5 \times 10^{4}$ and $1.2 \times 10^{5} L_{\odot}$ can be considered as RSGs only if their amplitude of variation in the optical $I$ band was less than $0.45 \mathrm{mag}$. This selection implied that seven objects in the Groenewegen sample were kept (marked with "s" 
Table 3. Red supergiants of Reid et al. (1990).

\begin{tabular}{lcrrr}
\hline \hline Name & $\alpha, \delta(\mathrm{J} 2000)$ & $L$ & $\dot{M}$ & $r$ \\
\hline HV 12501 & $045541.83-692624.3$ & 132000 & 157 &.. \\
HV 12420 & $045731.52-700859.2$ & 110000 & 79 &.. \\
HV 2255 & $045743.32-700850.3$ & 251000 & 196 & 37 \\
HV 888 & $050414.14-671614.4$ & 328000 & 134 & 37 \\
HV 894 & $050533.50-703347.0$ & 106000 & 86 &.. \\
HV 2360 & $051246.37-671937.9$ & 161000 & 135 &. \\
HV 916 & $051449.74-672719.8$ & 148000 & 96 & 37 \\
HV 2532 & $052627.39-691055.9$ & 113000 & 49 &.. \\
HV 963 & $052734.35-665330.0$ & 100000 & 56 & 31 \\
HV 5854 & $052815.41-665852.7$ & 97000 & 23 &.. \\
HV 2586 & $052934.43-665528.3$ & 71000 & 50 &.. \\
HV 996 & $053235.61-675508.9$ & 127000 & 172 & 26 \\
HV 12437 & $053307.61-664805.7$ & 82000 & 65 &.. \\
HV 2700 & $053518.92-670219.6$ & 89000 & 42 &.. \\
HV 1004 & $053625.46-665538.4$ & 141000 & 110 &.. \\
\hline
\end{tabular}

Notes. The columns provide the object name, J2000 coordinates as for 2MASS names, luminosities in $L_{\odot}$, mass-loss rates in units of $10^{-6} M_{\odot} \mathrm{yr}^{-1}$, and the ratios $r$ with mass-loss rates from Groenewegen et al. (2009) for common objects.

Table 4. Red supergiants of van Loon et al. (2005). Same columns as in Table 3.

\begin{tabular}{lcrrr}
\hline \hline Name & $\alpha, \delta(\mathrm{J} 2000)$ & $L$ & $\dot{M}$ & $r$ \\
\hline WOH-G64 & $045510.48-682029.8$ & 490000 & 1200 & 1.6 \\
HV 12501 & $045541.83-692624.3$ & 145000 & 15 &.. \\
HV 888 & $050414.14-671614.4$ & 269000 & 37 & 10 \\
HV 2360 & $051246.37-671937.9$ & 129000 & 30 &.. \\
HV 916 & $051449.74-672719.8$ & 148000 & 35 & 13 \\
HV 2561 & $052828.86-680707.9$ & 179000 & 49 & 16 \\
HV 5870 & $052903.48-690646.2$ & 95000 & 23 & 8 \\
SP77-46-44 & $052942.21-685717.4$ & 295000 & 52 & 37 \\
HV 986 & $053109.28-672554.9$ & 132000 & 20 &.. \\
HV 996 & $053235.61-675508.9$ & 117000 & 63 & 10 \\
\hline
\end{tabular}

after their names in the first column of Table 5) and nine were rejected.

Tables 3-5 include names, J2000 coordinates, luminosities, mass-loss rates (scaled as explained above and in units of $10^{-6} M_{\odot} \mathrm{yr}^{-1}$ ). The last column in Tables 3 and 4 is the ratio of the $\dot{M}$ from Reid et al. (or from van Loon et al.) and the $\dot{M}$ from Groenewegen et al. (2009) for objects belonging to both samples.

Figure 5 shows these scaled $\dot{M}$ as a function of $L$ from the three works mentioned above. We also show the de Jager rate for 4000 and $3500 \mathrm{~K}$ exactly as for previous plots. It is obvious that there is a difference between the recent rates from Groenewegen sample on one hand (the median rate is $2.3 \times 10^{-6} M_{\odot} \mathrm{yr}^{-1}, 30$ RSGs represented by circles), and, on the other hand, the Reid sample (median $9.0 \times 10^{-5} M_{\odot} \mathrm{yr}^{-1} ; 15$ crosses) or the van Loon sample (median $3.6 \times 10^{-5} \quad M_{\odot} \mathrm{yr}^{-1} ; 10$ small squares). One can also consider the objects in common (see Tables 3 and 4): these objects indicate that the Reid mass-loss rates are $\sim 35$ times higher than those of Groenewegen, and that the van Loon massloss rates are $\sim 10$ times higher than those of Groenewegen. One notes however that for WOH-G64 the $\dot{M}$ from van Loon and Groenewegen agree reasonably well (within a factor 1.6). In contrast, for SP77-46-44 (alias W60 A27), they disagree by a factor 37.

There are two assumptions in Reid et al. (1990) that can explain (at least in part) why their rates are so high. First, there
Table 5. Red supergiants of Groenewegen et al. (2009), with names, J2000 coordinates, luminosities, and mass-loss rates in units of $10^{-6} M_{\odot} \mathrm{yr}^{-1}$.

\begin{tabular}{lcrr}
\hline \hline Name & $\alpha, \delta(\mathrm{J} 2000)$ & $L$ & $\dot{M}$ \\
\hline HV 2236 & $044922.46-692434.4$ & 120000 & 2.8 \\
MSX LMC 1189 & $045503.07-692912.7$ & 139000 & 6.7 \\
WOH-G64 & $045510.48-682029.8$ & 440000 & 764 \\
MSX LMC 1204 & $045516.05-691912.1$ & 204000 & 0.81 \\
MSX LMC 1318 s & $045533.54-692459.3$ & 61000 & 1.3 \\
HV 2255 & $045743.31-700850.3$ & 143000 & 5.3 \\
HV 888 & $050414.13-671614.3$ & 337000 & 3.6 \\
HV 916 & $051449.72-672719.7$ & 250000 & 2.6 \\
WOH-S264 & $052419.32-693849.3$ & 215000 & 4.5 \\
MSX LMC 549 & $052611.35-661211.1$ & 132000 & 2.0 \\
MSX LMC 575 & $052622.18-662128.5$ & 163000 & 0.20 \\
MSX LMC 589 & $052634.80-685140.0$ & 269000 & 1.7 \\
HV 963 s & $052734.35-665330.0$ & 115000 & 1.8 \\
HV 2561 & $052828.86-680707.8$ & 139000 & 3.0 \\
HV 5870 s & $052903.48-690646.2$ & 79000 & 2.8 \\
W60 A27 & $052942.21-685717.3$ & 280000 & 1.4 \\
MSX LMC 810 & $053020.67-665301.8$ & 197000 & 1.1 \\
MSX LMC 587 & $053104.18-691903.0$ & 218000 & 2.3 \\
W60 D22 & $053110.64-663531.6$ & 163000 & 0.66 \\
MSX LMC 839 & $053136.81-663007.6$ & 255000 & 2.2 \\
HV 996 & $053235.61-675508.9$ & 132000 & 6.6 \\
MSX LMC 791 s & $053524.52-690403.4$ & 84000 & 0.89 \\
MSX LMC 870 & $053528.32-665602.4$ & 218000 & 2.3 \\
MSX LMC 891 & $053555.22-690959.4$ & 265000 & 3.1 \\
W60 A72 s & $053833.97-692031.7$ & 115000 & 84 \\
W60 A78 & $053932.33-693450.0$ & 223000 & 0.79 \\
MSX LMC 897 & $054043.75-692158.1$ & 245000 & 1.0 \\
MSX MC 939 s & $054048.50-693336.1$ & 109000 & 4.4 \\
HV 1017 s & $054059.20-691836.2$ & 102000 & 4.3 \\
HV 2834 & $054413.73-661644.5$ & 215000 & 1.1 \\
\hline & & &
\end{tabular}

is probably an overestimate of the flux averaged wavelength: Reid et al. (1990) take $\lambda_{10}=1$, which is not reached even by NML Cyg (see Table 2). For the optical RSGs making the Reid sample, $\lambda_{10}$ is likely close to 0.2 only. Secondly, Reid et al. (1990) overestimate the $f_{60} / f_{25}$ IRAS flux ratio used to obtain $f_{60}$. They take 0.50 instead of $\sim 0.20$. A ratio of $0.23 \pm 0.03$ is reached only by dusty Galactic RSGs (VX Sgr: 0.19, VY CMa: 0.22, PZ Cas: 0.24, NML Cyg: 0.26). Consequently the Reid et al. mass-loss rates have to be decreased by a factor $\sim 12$.

Concerning the van Loon rates, the reasons why they are generally higher than those of Groenewegen are not clear. With Spitzer data, small infrared excesses become measurable and are more accurate, while this was not the case with MSX, ISO, or IRAS data available for the van Loon et al. work. Therefore, there may be a bias in favour of large $\dot{M}$ in the van Loon sample compared to the Groenewegen sample. Another possibility is that the higher spatial resolution of Spitzer allows to avoid source confusion and contaminating sources. This may be in particular the case for SP77-46-44. For this source, the IRAS fluxes at 12 and $25 \mu \mathrm{m}$ are 0.26 and $0.18 \mathrm{Jy}$, while Spitzer photometry (from Bonanos et al. 2009) indicates $0.19 \mathrm{Jy}$ at $12 \mu \mathrm{m}$ (through interpolation from the $8 \mu \mathrm{m}$ and $24 \mu \mathrm{m}$ fluxes) and $0.11 \mathrm{Jy}$ at $25 \mu \mathrm{m}$. So these fainter Spitzer fluxes suggest a smaller infrared excess and may go some way to explain the large discrepancy for this object.

Finally, if we consider the rates by Groenewegen et al. (2009) and their positions with respect to the de Jager lines (see Fig. 5), one can note a rather good agreement for 12 objects with luminosities lower than $1.6 \times 10^{5} L_{\odot}$ (with the exception of W60-A72 


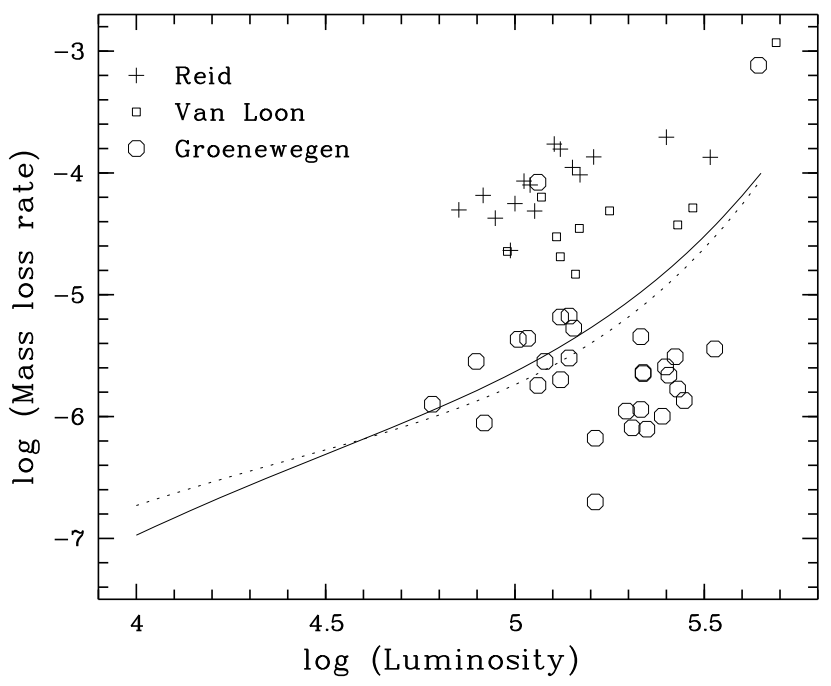

Fig. 5. Mass-loss rates plotted as a function of luminosity for the LMC RSGs. Three samples are plotted: Reid et al. (1990) (15 small crosses), van Loon et al. (2005) (10 small squares), and Groenewegen et al. (2009) (30 circles). The de Jager rates are represented as in previous figures by solid and dotted lines for $4000 \mathrm{~K}$ and $3500 \mathrm{~K}$, respectively.

at $L=115000 L_{\odot}, \dot{M}=8.4 \times 10^{-5} M_{\odot} \mathrm{yr}^{-1}$; in Fig. 5 this object is the circle located in the middle of Reid's plus signs). But at higher luminosities, the Groenewegen rates are below the dJ88 lines by an average factor $\sim 8$ (13 objects), with the exception of WOH-G64 where the de Jager rate is $\sim 10$ times lower than observed. It is also remarkable that at high luminosities, two objects from the van Loon sample are quite close to the dJ88 line.

\section{Discussion}

The goal of this paper is to see whether the de Jager prescription is still applicable in view of new results obtained since 1988 and concerning the RSGs and their winds. One important fact is that for only eight RSGs in the solar neighbourhood the direct detection of the circumstellar gas is available. In all other cases, only dust can be seen. We find that for these eight RSGs, the mass-loss rate estimated from observations of the gas is in most cases within a factor of 4 of the de Jager prescription for $L<2 \times 10^{5} L_{\odot}$ (Fig. 2). For higher luminosity it is a factor of 10 . These deviations have to be considered in light of the following characteristics of the RSG winds. The winds are changing with time (timescale $\sim 10^{2}-10^{4}$ years), and the rate of mass loss and the expansion velocity vary. This is proved by high-resolution spectroscopy of the circumstellar absorption lines, which display discrete velocity components (on $\mu$ Cep, Bernat 1981), by direct imagery ( $\alpha$ Ori, Plez $\&$ Lambert 2002) or by study of the CO millimetric lines (VY CMa, Decin et al. 2006). Also, the winds are often not spherically symmetrical, as revealed by some CO line profiles (PZ Cas, Woodhams 1993) or direct imagery (HST imaging of NML Cyg, Schuster et al. 2006). Given these properties, the measurements of $\dot{M}$ with often limited data and simple modelling are necessarily poor in accuracy and do not reflect an average mass-loss rate. Therefore, the agreement with the de Jager prescription seems acceptable.

For other, much more numerous RSGs, the gas is not detectable and only dust can be seen through its infrared radiation. When Jura's formula is adopted for deriving $\dot{M}$, the resulting $\dot{M}$ also agree with de Jager lines, except for some rare stars like $\mu$ Cep, which has a very low dust content. In general, the agreement is within a factor of 4 (Fig. 2). Part of this deviation may be caused by a natural scatter of $\dot{M}$ at a given luminosity (see the characteristics of winds mentioned above) and another part may be owing to the use of Jura's formula.

\subsection{Verification of the Jura's formula}

In order to check Jura's formula, we have considered the work by Verhoelst et al. (2009) who exploit ISO-SWS spectra (in addition to the full spectral energy distribution) and use state-of-the-art dust modelling. In particular, they include several types of grains such as melilite, olivine, alumine, carbon and FeO. Together with results concerning the dust condensation sequence in RSGs, they derive mass-loss rates. We took into account their assumptions on distance, expansion velocity and gas-to-dust mass ratio and we applied appropriate scaling, so that we can compare their results to ours ( $\dot{M}_{60 \mu \mathrm{m}}$ in Table 2$)$. We find that their $\dot{M}$ are 0.57 , $0.88,1.20$ and 1.9 times ours, for $\alpha$ Ori, S Per, PZ Cas and $\mu$ Cep, respectively. In conclusion, the agreement with Jura's formula is at most a factor of 2, which is very good, and there is no particular reason to have doubts on this formula.

\subsection{Comparison with other mass-loss prescriptions}

In Sect. 2, we mentioned in Fig. 1 a few mass-loss prescriptions. After our analysis of previous sections, we can reconsider this point. Concerning the RSGs in the solar vicinity, all observed mass-loss rates (from dust or gas observations) are well below the rate by Salasnich et al. (1999). For example, $\alpha$ Ori and $\alpha$ Sco are located at $L \approx 60000 L_{\odot}$, and their $\dot{M}$ are $\sim 1.5 \times 10^{-6} M_{\odot} \mathrm{yr}^{-1}$. The Salasnich rate would give $3.4 \times 10^{-5} \quad M_{\odot} \mathrm{yr}^{-1}, 20$ times larger. Because they are based on mass-loss rates of Reid et al. which we have shown are overestimated, the Salasnich rate and the Feast rate are too large. The Reimers rate is as good as the dJ88 rate for objects with $L \lesssim 2 \times 10^{5} L_{\odot}$, but this is not the case for higher luminosity objects like NML Cyg or VY CMa.

\subsection{Comparison with the van Loon formalism}

It is appropriate to consider here the mass-loss formulation of van Loon et al. (2005). These authors derived a prescription for mass-loss rates of AGB stars and red supergiants. It is based on the study of evolved stars in the LMC, but is also valid for Galactic AGB stars and RSGs. An important feature of this prescription is that it is applicable only to dusty stars. Unfortunately, the dusty character of a circumstellar envelope is not possible to be derived from the basic stellar parameters, i.e. $L, M$ and $T_{\text {eff }}$. Nevertheless, one can compare the results of this prescription to the de Jager rate. van Loon et al. (2005) derived effective temperatures from spectral classifications. Luminosities and mass-loss rates were determined by modelling the spectral energy distribution. The van Loon prescription is:

$$
\dot{M}=2.50 \times 10^{-5}\left(L / 10^{5}\right)^{1.05}(T / 3500)^{-6.3} .
$$

This prescription is shown in Fig. 6 along with the de Jager lines. Both are drawn for $T_{\text {eff }}$ of 4000,3500 and $3000 \mathrm{~K}$. van Loon et al. (2005) have found that their recipe overestimates massloss rates for Galactic "optical" RSGs. We can see that clearly in Fig. 6, where the van Loon lines are well above the de Jager lines on which we found that most of the Galactic RSG are located (Figs. 2 and 3). However, the de Jager rate and the van Loon rate fairly agree with each other for the highest luminosities. Also, it 


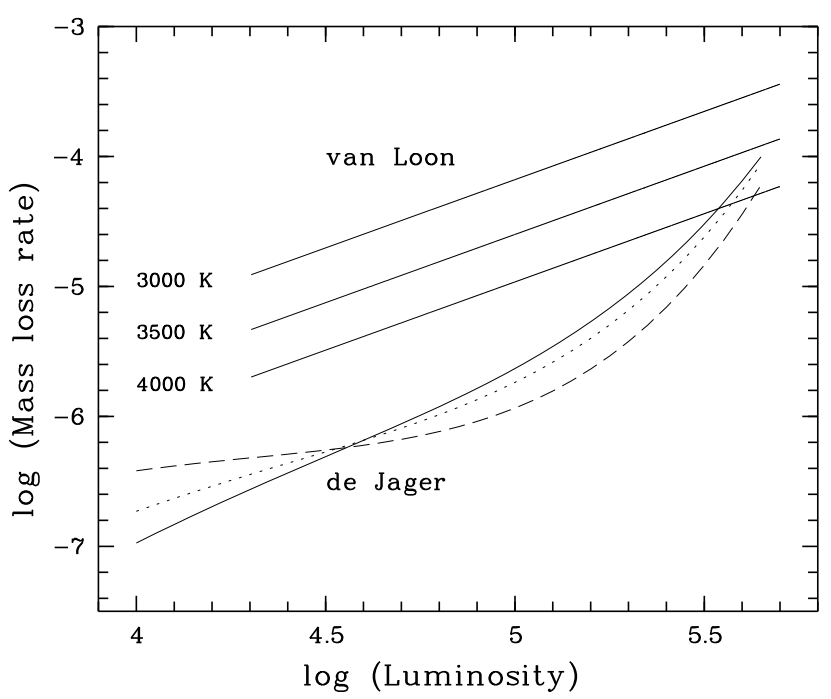

Fig. 6. Mass-loss rate prescription of van Loon et al. (2005) and de Jager (1988) with $\dot{M}$ plotted as a function of luminosity and for three values of $T_{\text {eff }}$. The de Jager lines are solid, dotted and dashed for 4000, 3500 and $3000 \mathrm{~K}$ respectively.

is worth noting that the van Loon et al. prescription offers a significant spread of $\dot{M}$ for a given luminosity. The spread allowed by the deJ88 prescription is clearly very small.

We have collected in Table 6 the mass-loss rates for the eight stars possessing an $\dot{M}_{\text {gas }}$ based on observation of the gas (from Table 2). In this table, the $T_{\text {eff }}$ values are taken from Lambert et al. (1984) for $\alpha$ Ori, from LM05 for S Per, PZ Cas, and $\mu$ Cep, from van Loon et al. (2005) for VY CMa, NML Cyg and VX Sgr, and from Kudritzki \& Reimers (1978) for $\alpha$ Sco. Column 5 of this table gives the de Jager rates, and Col. 6 gives the van Loon rates (in Col. 6, the numbers in parenthesis concern "optical" RSGs and are just given for information). The ratios between van Loon rates and the observed rates are 2.8, 5.1, 2.2, 0.85 and 7.2 for S Per, PZ Cas, VY CMa, NML Cyg, and VX Sgr, respectively. By considering the median of these numbers, the van Loon rates are $\sim 2.8$ times too large on average. For comparison, the ratios between de Jager rates and observed rates for the same stars are $0.20,0.78,0.45,0.15$ and 2.9 . The median is 0.45 so that, the de Jager rates are $\sim 2.2$ times too small on average. In conclusion, the de Jager and van Loon rates are almost equally accurate (or inaccurate), but one (de Jager) underestimates and the other (van Loon) overestimates the observed rates. The de Jager formalism is closer to the observed rates for non-dusty RSGs, although it is poor in the case of $\mu$ Cep (off by an order of magnitude).

\subsection{The behaviour of the de Jager rate with effective temperature}

Figure 6 shows in particular the temperature dependance for the van Loon and de Jager prescriptions. It is surprising that for $\log L \gtrsim 4.5$, the $\dot{M}$ of de Jager gets smaller for decreasing $T_{\text {eff }}$. We cannot explain this behaviour in view of the data concerning the 15 RSGs that dJ88 used when deriving their formula (these data are listed in Appendix A). In particular, VY CMa has the largest mass-loss rate of their small sample $\left(2.4 \times 10^{-4 \sim} M_{\odot} \mathrm{yr}^{-1}\right)$ and its temperature adopted by dJ88 is low, $T_{\text {eff }}=2840 \mathrm{~K}$. In addition, the weight assigned to this star by $\mathrm{dJ} 88$ is considerably larger (60) than the weight of any other RSG in the sample
Table 6. Red supergiants with an observed mass-loss rate derived from observations of the gas $\left(\dot{M}_{\text {gas }}\right)$.

\begin{tabular}{lccrcr}
\hline \hline $\begin{array}{l}\text { Name } \\
(1)\end{array}$ & $\begin{array}{c}L \\
(2)\end{array}$ & $\begin{array}{c}T_{\text {eff }} \\
(3)\end{array}$ & $\begin{array}{c}\dot{M}_{\text {gas }} \\
(4)\end{array}$ & $\begin{array}{c}\dot{M}(\mathrm{deJ}) \\
(5)\end{array}$ & $\begin{array}{r}\dot{M}(\mathrm{VL}) \\
(6)\end{array}$ \\
\hline$\alpha$ Ori & 56000 & 3800 & 1.5 & 1.0 & $(8)$ \\
$\alpha$ Sco & 71000 & 3550 & 1.0 & 1.2 & $(16)$ \\
S Per & 86000 & 3500 & 7.5 & 1.5 & 21 \\
PZ Cas & 193000 & 3600 & 8.3 & 6.5 & 42 \\
VY CMa & 295000 & 3435 & 40.0 & 18 & 88 \\
NML Cyg & 320000 & 3310 & 140.0 & 21 & 120 \\
VX Sgr & 340000 & 3575 & 11.0 & 32 & 79 \\
$\mu$ Cep & 410000 & 3700 & 5.0 & 67 & $(78)$ \\
\hline
\end{tabular}

Notes. Column 5 is the de Jager rate, and Col. 6 is the rate with the van Loon recipe, which is applicable only for dusty stars (rates in parentheses should not be considered). Mass-loss rates are given in units of $10^{-6} M_{\odot} \mathrm{yr}^{-1}$.

(weights $\sim 1-4)$. Yet, the de Jager rate decreases for cooler temperatures. A possible explanation of this peculiar $T_{\text {eff }}$ behaviour is the fact that the fit of the data for the dJ88 rate was made globally on all stars over the H-R diagram and not just "locally" on the RSGs.

\subsection{Is the scatter of $\dot{M}$ at a given luminosity due to effective temperature?}

An interesting question is whether the deviation of the measured $\dot{M}$ from the dJ88 rate is correlated with effective temperature. Thanks to the work of LM05, $T_{\text {eff }}$ is known for the 27 first RSGs of Table 1 (from V589 Cas to TZ Cas). $T_{\text {eff }}$ is also known for the 8 stars of Table 6 . We adopt the infrared-based $\dot{M}$ from Table 1, but prefer when possible $\dot{M}_{\text {gas }}$ from Table 6. Figure 7 (panel a) plots the ratio (in $\log$ ) of measured mass-loss rate to the de Jager rate. It shows a slight tendency of $\dot{M}$ decreasing with increasing $T_{\text {eff. }}$ The standard deviation is 0.410 when all 31 stars are considered and it can be seen that 6 stars (of 31) have ratios larger than 4 or smaller than $1 / 4$. Changing the de Jager rate by adding a $T_{\text {eff }}^{-\alpha}$ factor would slightly reduce this scatter. Values of $\alpha$ between 6 and 10 are suitable. We show in panel $b$ the resulting situation for $\left(T_{\text {eff }} / 3600\right)^{-10}$. The standard deviation is 0.367 for all objects included. It is 0.296 if $\mu$ Cep and V396 Cen are omitted, and all other objects are within a factor of 4 of agreement. V396 Cen has a poor quality $60 \mu \mathrm{m}$ flux, coded $\mathrm{F}$ in the IRAS point source catalog, and presents an anormally low $f_{60} / f_{25}$ flux ratio. Therefore its position in Fig. 7 is plausibly explained by an underestimated $60 \mu \mathrm{m}$ flux.

\subsection{The RSGs in the Magellanic Clouds and the dependence on metallicity}

We have seen that the mass-loss rates in the LMC from Groenewegen et al. do not follow fully the de Jager rate. For $L$ between 63000 and 160000 , there is reasonable agreement, but at larger $L$, the de Jager rate is off (too large) by a factor of $\sim 8$. Recently, Bonanos et al. (2010) have also considered the mass-loss rates of RSGs in the LMC and SMC. Their sample of RSGs ( $\sim 100$ sources in the LMC, 34 in the SMC) is taken from a list of luminous objects having optical spectral classification and photometry from 0.3 to $24 \mu \mathrm{m}$ obtained during the Spitzer SAGE survey of the Magellanic Clouds (Meixner et al. 2006). Bonanos et al. (2010) estimate mass-loss with a $\dot{M}$ versus the $K-$ [24] relation derived from model dusty envelopes. 

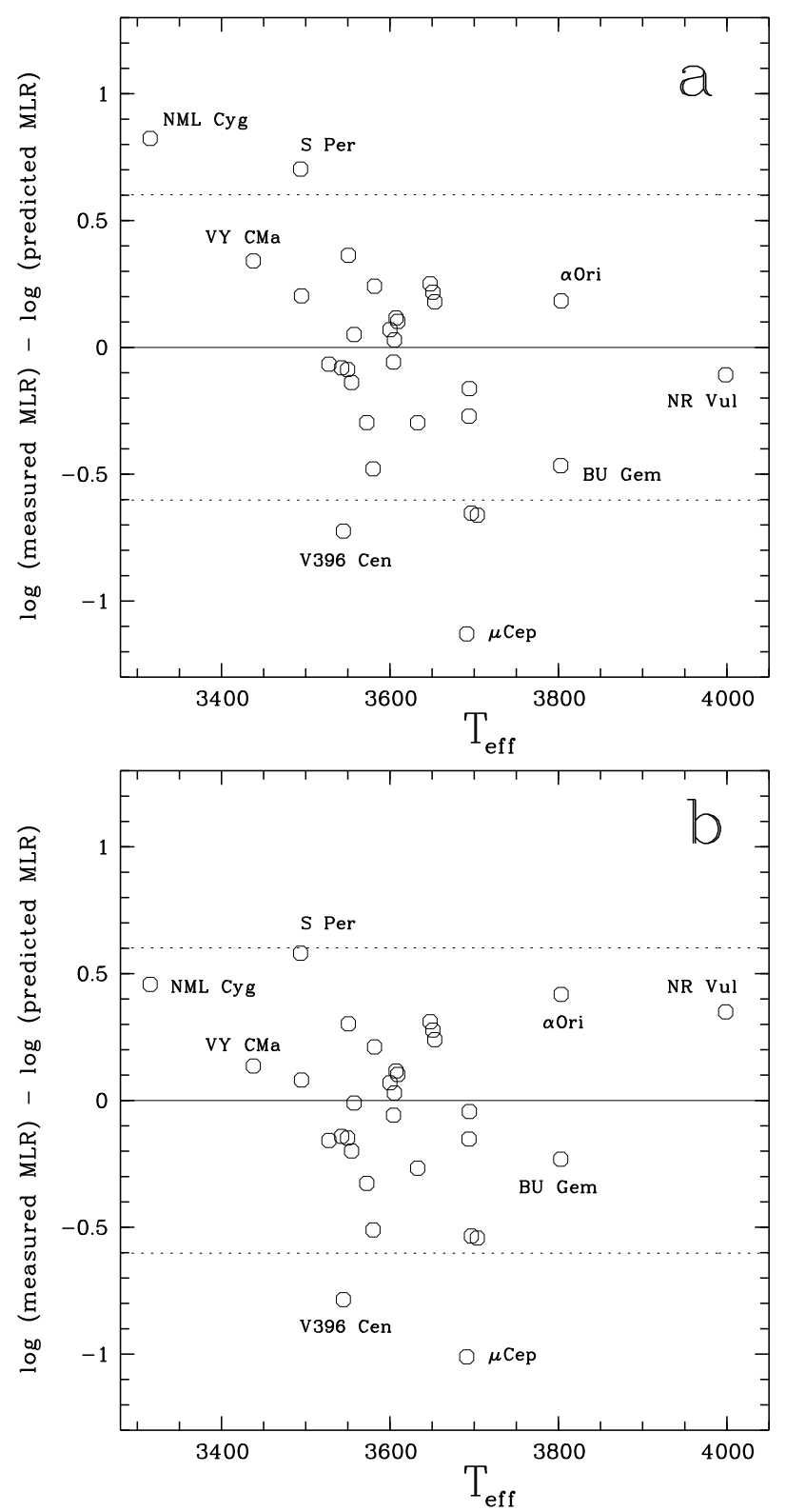

Fig. 7. Panel a): discrepancies between measured mass-loss rates (MLR) and mass-loss rates predicted by the de Jager prescription, plotted versus $T_{\text {eff }}$, for Galactic red supergiants. The horizontal dotted lines indicate discrepancies by factors 4 and $1 / 4$. Panel $\mathbf{b}$ ): same as panel a) when the de Jager rate is multiplied by $\left(T_{\text {eff }} / 3600\right)^{-10}$.

From the data plotted in their Fig. 17, which show important scatter at a given luminosity, we derive median mass-loss rates in several bins in $L$. The 5 bins for the LMC have respectively $15,24,28,25$ and 5 data points. The 4 bins for the SMC have $6,10,11$ and 7 data points. The resulting $\dot{M}$ versus $L$ relations are shown in Fig. 8 (histograms). Both the SMC and LMC data suggest that $\dot{M}$ increases with luminosity if $\log L$ is less than $\sim 5.3$, as already concluded by Bonanos et al. (2010). It can be noted that the LMC bin with the highest luminosity suggests that $\dot{M}$ could saturate, but the low number of points makes it difficult to be sure of that (see these 5 points at the highest luminosity in Fig. 17 of Bonanos et al. 2010). However, we note that this is quite consistent with our Fig. 5 and its high- $L$, low- $\dot{M}$ group of stars, as mentioned above.

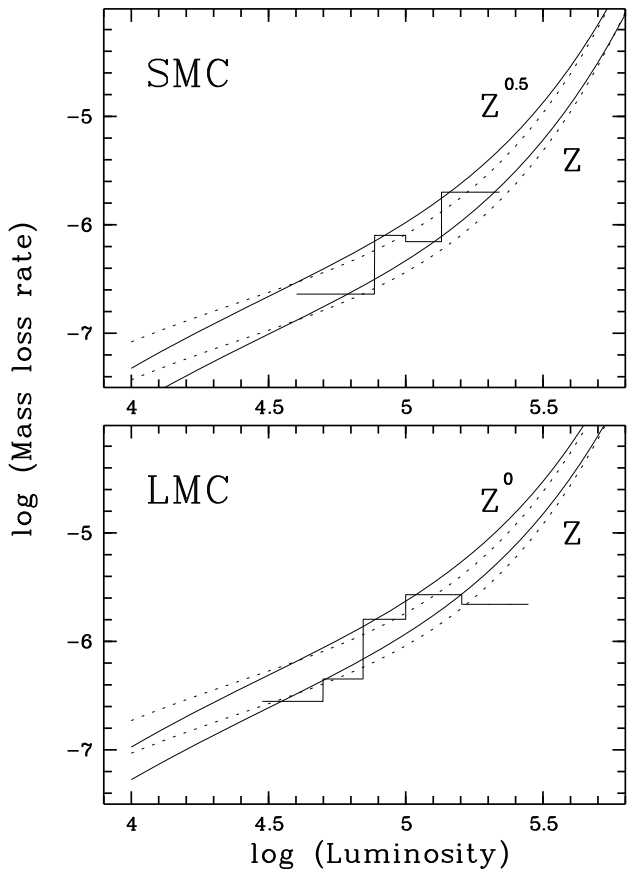

Fig. 8. Mass-loss rates versus luminosity for Magellanic Clouds. The (median) data of Bonanos et al. (2010) are represented by histograms (see text). The solid and dotted lines show the dJ88 rates for 3500 and $4000 \mathrm{~K}$ scaled for various metallicity dependences, as indicated in the panels.

In order to investigate how the RSG mass-loss rates depend on metallicity, we can compare the median rates obtained above with the dJ88 prescription scaled by various $Z$ values. Here we adopt $Z(\mathrm{LMC})=Z_{\odot} / 2$, and $Z(\mathrm{SMC})=Z_{\odot} / 5$. Figure 8 shows the de Jager lines for warm effective temperatures which are suitable for the Clouds. It shows that, for the SMC, the observed rates are between the de Jager rate scaled by $Z^{0.5}$ and $Z$, and a $Z^{0.7}$ scaling would fit the data quite well. As for the LMC, the situation is less clear because the metallicity decrease is only a factor 2 with respect to solar. If we omit the most luminous bin, a $Z^{0.7}$ scaling is not unreasonable, but a $Z^{0.5}$ is also possible.

\section{Conclusions}

The de Jager prescription used in evolution codes for RSGs was established in 1988 when the mass-loss rates of stars located over all the $H$-R diagram were fitted as a function of $L$ and $T_{\text {eff }}$. The fit was not specifically made for this type of stars, and the goal of this work was to compare the de Jager rate to estimates of $\dot{M}$ based on data acquired after 1988. Our main conclusions are the following:

1. When Jura's formula is adopted to get an observational estimate of $\dot{M}$ based on the IRAS infrared excess at $60 \mu \mathrm{m}$ and supposing a gas-to-dust ratio of 200 (corresponding to complete condensation of refractory material), it is found that the Galactic RSGs are satisfactorily distributed around the de Jager line. The deviation from de Jager rate is in general less than a factor of 4 .

2. Only eight Galactic RSGs have their circumstellar gas detected, i.e. through detection of $\mathrm{HI}$ or $\mathrm{CO}$ emission, or other methods permitting to estimate $\dot{M}$ from observation of the gas. For these stars, $\dot{M}$ agrees reasonably well with the de Jager rate. The most discrepant cases are found at 
high luminosity, where the de Jager prescription underestimates $\dot{M}$ of NML Cyg by a factor of 7, but overestimates $\dot{M}$ for $\mu$ Cep by a factor of 10 (see Table 3).

3. The Spitzer data from Bonanos et al. (2010) show that the mass-loss rates of RSGs in the Small Magellanic Cloud agree on average with the de Jager rate scaled for metallicity by $\left(Z / Z_{\odot}\right)^{0.7}$.

4. A similar behaviour is less clear with RSGs of the Large Magellanic Cloud. For luminosities lower than $1.6 \times 10^{5} L_{\odot}$, the median mass-loss rates roughly follow the de Jager prescription and its increasing trend with luminosity. These measured rates are also roughly compatible with the $Z^{0.7}$ scaling found for the SMC. However, for higher luminosities, one finds that the mass-loss rates level off at a rate of $\sim 3 \times 10^{-6} M_{\odot} \mathrm{yr}^{-1}$ and are smaller by a factor of $\sim 8$ than the (unscaled) dJ88 rate (with the exception of WOH-G64).

5. Finally, although the dJ88 prescription presents some odd aspects that can be criticized (e.g., its behaviour with $T_{\text {eff }}$ ), it passes several tests fairly satisfactorily (listed 1, 2, 3, and part of 4 above). Therefore, we think it more appropriate to keep it unchanged in the stellar evolution models, but to apply a $Z^{0.7}$ factor for metallicity dependence. The LMC massloss saturation issue needs to be clarified. We hope to further contribute to that subject in the future.

Acknowledgements. We thank our referee, J. Th. van Loon, for many remarks that considerably improved the paper. N.M. warmly thanks Olivier Richard for his help concerning computers, and Tim Kendall and Astrid Peter for their help concerning English. N.M. also thanks Raphael Hirschi for discussions about the Geneva code. We acknowledge the use of the database produced by the Centre de Données de Strasbourg (CDS). This research was partly supported by the French National Research Agency (ANR) through program number ANR-06-BLAN-0105.

\section{References}

Bennett, P. D. 2010, in Hot and Cool: Bridging Gaps in Massive Star Evolution, ed. C. Leitherer, P. D. Bennett, P. W. Morris, \& J. van Loon, ASP Conf. Ser., 181

Bernat, A. 1981, ApJ, 246, 184

Blöcker, T., Balega, Y., Hofmann, K.-H., \& Weigelt, G. 2001, A\&A, 369, 142

Bonanos, A. Z., Massa, D. L., Sewilo, M., et al. 2009, ApJ, 138, 1003

Bonanos, A. Z., Lennon, D. J., Köhlinger, F., et al. 2010, AJ, 140, 416

Bowers, P. F., \& Knapp, G. R. 1987, ApJ, 315, 305

Cardelli, J. A., Clayton, G. C., \& Mathis, J. S. 1989, ApJ, 345, 245

Chen, X., Shen, Z.-Q., \& Xu, Y. 2007, Chin. J. Astron. Astrophys., 7, 531

Chevalier, R. A., Fransson, C., \& Nymark, T. K. 2006, ApJ, 641, 1029

Choi, Y. K., Hirota, T., Honma, M., et al. 2008, PASJ, 60, 1007

Decin, L., Hont, S., de Koter, A., et al. 2006, A\&A, 456, 549

de Jager, C., Nieuwenhuijzen, H., \& van der Hucht, K. A. 1988, A\&ASS, 72, 259 (dJ88)

de Wit, W. J., Oudmaijer, R. D., Fujiyoshi, T., et al. 2008, ApJ, 685, L75

Eggenberger, P., Meynet, G., Maeder, A., et al. 2008, Astrophys. Space Sci., 316, 43

Feast, M. W. 1992, in Instabilities in Evolved Super and Hypergiants, ed. C. de Jager, \& H. Nieuwenhuijzen (Amsterdam: North Holland), 18

Gehrz, R. D., \& Woolf, N. J. 1971, ApJ, 165, 285

Gezari, D. Y., Schmitz, M., Pitts, P. S., \& Mead, J. M. 1993, Catalog of Infrared Observations, third edition, NASA Reference Publication 1294

Glassgold, A. E., \& Huggins, P. J. 1986, ApJ, 306, 60

Groenewegen, M. A. T., Sloan, G. C., Soszyński, I., \& Petersen, E. A. 2009, A\&A, 506, 1277
Guilain, Ch., \& Mauron, N. 1996, A\&A, 314, 585

Gustafsson, B., Eriksson, K., Kiselman, D., Olander, N., \& Olofsson, H. 1997, A\&A, 318, 535

Habing, H. J., Tignon, J., \& Tielens, A. G. G. M. 1994, A\&A, 286, 523

Hagen, W. 1982, PASP, 94, 835

Harper, G., Brown, A., \& Guinan, E. F. 2008, ApJ, 135, 1430

Huggins, P. J., Bachiller, R., Cox, P., \& Forveille, T. 1994, ApJ, 424, L127

Humphreys, R. M. 1978, ApJSS, 38, 309

Josselin, E., \& Lançon, A. 2009 [arXiv: 0911 . 0840]

Josselin, E., \& Plez, B. 2007, A\&A, 469, 671

Josselin, E., Blommaert, J. A. D. L., Groenewegen, M. A. T., Omont, A., \& Li, F. L. 2000, A\&A, 357, 225 (JB00)

Jura, M., \& Kleinmann, S. G. 1990, ApJS, 73, 769 (JK90)

Kemper, F., Stark, R., Justtanont, K., et al. 2003, A\&A, 407, 609

Keene, J., Young, K., Phillips, T. G., \& Büttgenbach, Th. H. 1993, ApJ, 415, L131

Kiss, L. L., Szabó, G. M., \& Bedding, T. R. 2006, MNRAS, 372, 1721

Knapp, G. R., Phillips, T. G., Leighton, R. B., et al. 1982, ApJ, 252, 616

Knapp, G. R., Sutin, B. M., Phillips, T. G., et al. 1989, ApJ, 336, 822

Kudritzki, R. P., \& Reimers, D. 1978, A\&A, 70, 227

Lambert, D. L., Brown, J. A., Hinkle, K. H., \& Johnson, H. R. 1984, ApJ, 284, 223

Le Borgne, J.-F., \& Mauron, N. 1989, A\&A, 210, 198

Lee, T. A. 1970, ApJ, 162, 217

Levesque, E. M. 2009, The Physical Properties of Red Supergiants, to appear New Astron. Rev. [arXiv:0911.4720]

Levesque, E. M., Massey, P., Olsen, K. A. G., et al. 2005, ApJ, 628, 973 (LM05) Maeder, A., \& Meynet, G. 1989, A\&A, 210,155

Marshall, J. R., van Loon, J. T., Matsuura, M., et al. 2004, MNRAS, 355, 1348

Massey, P. 1998, ApJ, 501,153

Massey, P., \& Thompson, A. B. 1991, AJ, 101, 1408

Massey, P., Levesque, E. M., Plez, B., \& Olsen, K. A. G. 2008, Proc. IAU Symp., 250, 97

Massey, P., Silva, D. R., Levesque, E. M., et al. 2009, ApJ, 703, 420

Mauron, N. 1990, A\&A, 227, 141

Meixner, M., Gordon, K. D., Indebetouw, R., et al. 2006, AJ, 132, 2268

Nieuwenhuijzen, H., \& de Jager, C. 1990, A\&A 231, 134

Olofsson, H. 2004, Circumstellar Envelopes, in Asymptotic Giant Branch Stars, ed. H. J. Habing, \& H. Olofsson (New-York: Springer), 325

Plez, B., \& Lambert, D. L. 2002, A\&A, 386, 1009

Ramstedt, S., Schöier, F. L., Olofsson, H., \& Lundgren, A. A. 2008, A\&A, 487, 645

Reid, N., Tinney, Ch., \& Mould, J. 1990, ApJ, 348, 98

Reimers, D. 1975, Mem. Soc. Roy. Sci. Liège, 6e Série, 8, 369

Reimers, D., Hagen, H.-J., Baade, R., \& Braun, K. 2008, A\&A, 491, 229

Salasnich, B., Bressan, A., \& Chiosi, C. 1999, A\&A, 342, 131

Sanner, F. 1976, ApJSS, 32, 115

Schröder, K.-P., \& Cuntz, M. 2007, A\&A, 465, 593

Schuster, M. T., Humphreys, R. M., \& Marengo, M. 2006, AJ, 131, 603

Schuster, M. T., Marengo, M., Hora, J. L., et al. 2009, ApJ, 699, 1423

Smartt, S. J., Eldridge, J. J., Crockett, R. M., \& Maund, J. R. 2009, MNRAS, 395,1409

Smith, N., Hinkle, K., \& Ryde, N. 2009, ApJ, 137, 3558

Stancliffe, R. J., \& Eldridge, J. J. 2009, MNRAS, 396, 1699

Sylvester, R. J., Barlow, M. J., \& Skinner, C. J. 1994, MNRAS, 266, 640

Sylvester, R. J., Skinner, C. J., \& Barlow, M. J. 1998, MNRAS, 301, 1083

Vanbeveren, D., De Loore, C., \& Van Rensbergen, W. 1998, A\&AR, 9, 63

Vanbeveren, D., Van Bever, J., \& Belkus, H. 2007, ApJ, 662, L107

van Loon, J. T. 2010, in Hot and Cool: Bridging Gaps in Massive Star Evolution, ed. C. Leitherer, P. D. Bennett, P. W. Morris, \& J. Th. van Loon (San Francisco) ASP Conf. Ser., 425

van Loon, J. Th., Cioni, M.-R. L., Zijlstra, A. A., \& Loup, C. 2005, A\&A, 438, 273

Verhoelst T., van der Zypen, N., Hony, S., et al. 2009, A\&A, 498, 127

Woitke, P. 2007, in Why Galaxies Care about AGB Stars: Their Importance as Actors and Probes, ed. K. Kerschbaum, C. Charbonnel, \& R. F. Wing (San Francisco) ASP Conf Ser., 378, 156

Woodhams, M. 1993, in Massive Stars: Their Lives in the Interstellar Medium, ed. J. P. Cassinelli, \& E. B. Churchwell (San Francisco) ASP Conf. Ser., 35, 231

Pages 12 to 14 are available in the electronic edition of the journal at http: //www . aanda.org 


\section{Appendix A: List of red supergiants considered by de Jager et al. (1988)}

The list of RSGs considered by dJ88 are given in Table A.1. The data from dJ88 are the stellar effective temperature $T_{1}(\mathrm{~K})$, the luminosity $L_{1}\left(L_{\odot}\right)$ and the mass-loss rate $\dot{M}_{1}$ in $M_{\odot} \mathrm{yr}^{-1}$. The columns $T_{2}, L_{2}$ and $\dot{M}_{2}$ give the same quantities as derived in this work. Many mass-loss rates used by dJ88 $\left(\dot{M}_{1}\right)$ came from the exploitation and analysis of infrared flux measurements between 3.5 and $11.5 \mu \mathrm{m}$ (Gehrz \& Woolf 1971). The mass-loss rates of Hagen (1982) are derived from flux measurements at 20 and $25 \mu \mathrm{m}$. The mass-loss rates of Sanner (1976) are based on high-resolution spectral profiles of strong resonance lines. For objects with $\dot{M}_{1}$ based on several individual determinations (from four to nine determinations for $\mu$ Cep and $\alpha$ Ori, respectively), see the details in de Jager et al. (1988).

Table A.1. List and data for the RSGs considered by dJ88.

\begin{tabular}{lccccccc}
\hline \hline Object & $T_{1}$ & $T_{2}$ & $\log L_{1}$ & $\log L_{2}$ & $\log \left(\dot{M}_{1}\right)$ & $\log \left(\dot{M}_{2}\right)$ & Note \\
\hline TV Gem & 3560 & 3700 & 4.75 & 4.92 & -5.92 & -6.46 & $\mathrm{GW}$ \\
BU Gem & 3440 & 3800 & 5.15 & 4.93 & -5.68 & -6.23 & $\mathrm{GW}$ \\
$\alpha$ Ori & 3500 & 3810 & 4.87 & 4.75 & -6.02 & -5.82 & $(1)$ \\
$\alpha$ Sco & 3540 & 3550 & 4.74 & 4.85 & -6.08 & -6.00 & $(1)$ \\
RW Cyg & 3365 & 3600 & 5.20 & 5.16 & -5.30 & -5.50 & $\mathrm{GW}$ \\
VV Cep & 3175 & $\ldots$ & 5.13 & $\ldots$ & -6.89 & $\ldots$ & $\mathrm{GW}$ \\
CE Tau & 3400 & $\ldots$ & 4.55 & $\ldots$ & -6.62 & $\ldots$ & $(4)$ \\
$\mu$ Cep & 3400 & 3700 & 4.55 & 5.61 & -5.78 & -5.30 & $(1)$ \\
BC Cyg & 3155 & 3575 & 5.54 & $\ldots$ & -5.15 & $\ldots$ & $\mathrm{GW}(2)$ \\
BI Cyg & 3155 & 3575 & 5.38 & 5.09 & -5.15 & -5.33 & $\mathrm{GW}$ \\
VY CMa & 2840 & 3430 & 5.76 & 5.47 & -3.62 & -4.40 & $(1)$ \\
EV Car & 2930 & $\ldots$ & 5.74 & 5.83 & -6.00 & -4.90 & $(3)$ \\
S Per & 2810 & $\ldots$ & 5.66 & 4.93 & -4.57 & -5.16 & $\mathrm{GW}$ \\
UY Sct & 3000 & $\ldots$ & 5.44 & $\ldots$ & -5.22 & $\ldots$ & $\mathrm{GW}$ \\
AH Sco & 2800 & $\ldots$ & 5.62 & $\ldots$ & -6.00 & $\ldots$ & $(3)$ \\
NML Cyg & 2485 & 3309 & 5.80 & 5.50 & -4.18 & -3.85 & $(1)$ \\
\hline
\end{tabular}

Notes. GW indicates that $\dot{M}_{1}$ was taken by dJ88 from Gehrz \& Woolf (1971). (1) Indicates that $\dot{M}_{1}$ is the average of several determinations obtained with different methods. (2) BC Cyg has no IRAS fluxes at 25 and $60 \mu \mathrm{m}$ and was not included in this study. (3) $\dot{M}_{1}$ from Hagen (1982). (4) $\dot{M}_{1}$ from Sanner (1976). 


\section{Appendix B: Photometric data of the studied RSGs}

Table B.1 presents the magnitudes in the $U B V I J H K$ filters and the IRAS fluxes at 12,25 , and $60 \mu \mathrm{m}$ that were used to calculate the flux averaged wavelength $\lambda_{\mathrm{m}}$, and the luminosities $L$. If a magnitude in the above filters was not available, we used the following relations, which were established from the sample data:

$$
\begin{aligned}
& U \approx 0.62+0.78(B-V) \\
& I \approx V-0.80(V-J) \\
& L \approx K-0.27-0.119(K-[12]) \\
& f_{60} \approx 0.20 f_{25} \\
& f_{100} \approx 0.33 f_{60} .
\end{aligned}
$$

\begin{tabular}{|c|c|c|c|c|c|c|c|c|c|c|c|}
\hline Object & $U$ & $B$ & $V$ & $I$ & $J$ & $H$ & $K$ & $L$ & $f_{12}$ & $f_{25}$ & $f_{60}$ \\
\hline BD+60335 = V589 Cas & 13.94 & 11.49 & 9.15 & 5.03 & 4.06 & 3.01 & 2.49 & 2.16 & 12.74 & 8.67 & 3.61 \\
\hline $\mathrm{BD}+56512=\mathrm{BU}$ Per & 14.35 & 11.70 & 9.23 & 4.80 & 3.68 & 2.68 & 2.19 & 1.85 & 44.97 & 31.29 & 5.23 \\
\hline HD $14469=$ SU Per & 12.04 & 9.80 & 7.63 & 3.82 & 2.82 & 1.93 & 1.46 & 1.13 & 48.71 & 30.66 & 6.87 \\
\hline HD $14488=$ RS Per & 12.90 & 10.62 & 8.35 & 4.33 & 3.05 & 2.11 & 1.56 & 1.22 & 74.44 & 47.82 & 9.93 \\
\hline HD $14528=$ S Per & 14.51 & 11.88 & 9.23 & 4.48 & 2.95 & 1.84 & 1.12 & 0.55 & 339.40 & 233.20 & 40.59 \\
\hline HD $14826=$ V441 Per & 13.02 & 10.56 & 8.24 & 4.30 & 3.47 & 2.47 & 2.04 & 1.60 & 18.53 & 13.25 & 3.54 \\
\hline HD 936979 = YZ Per & 13.14 & 10.55 & 8.20 & 4.34 & 3.26 & 2.30 & 1.91 & 1.64 & 38.85 & 26.12 & 5.28 \\
\hline W Per & 14.80 & 12.24 & 9.62 & 4.75 & 3.09 & 2.00 & 1.57 & 1.51 & 90.58 & 78.86 & 14.87 \\
\hline $\mathrm{BD}+57647$ & 14.93 & 12.26 & 9.52 & 4.76 & 3.83 & 2.71 & 2.11 & 1.56 & 39.04 & 26.45 & 6.47 \\
\hline HD $37536=$ NO Aur & 10.52 & 8.30 & 6.21 & 3.01 & 2.12 & 1.13 & 0.97 & 0.51 & 43.49 & 22.89 & 5.12 \\
\hline$\alpha$ Ori & 4.36 & 2.29 & 0.40 & -2.47 & -2.93 & -3.73 & -4.01 & -4.43 & 4680.00 & 1740.00 & 299.00 \\
\hline HD $42475=$ TV Gem & 10.58 & 8.81 & 6.56 & 3.17 & 2.20 & 1.20 & 0.94 & 0.54 & 96.08 & 41.16 & 6.06 \\
\hline HD $42543=$ BU Gem & 11.11 & 8.63 & 6.39 & 3.07 & 2.23 & 1.28 & 0.98 & 0.62 & 78.20 & 47.59 & 10.50 \\
\hline CD-314916 = V384 Pup & 13.37 & 11.07 & 8.91 & 5.43 & 4.61 & 3.49 & 3.14 & 2.83 & 10.85 & 7.34 & 2.76 \\
\hline HD 90382 = CK Car & 12.00 & 9.66 & 7.45 & 3.55 & 2.63 & 1.71 & 1.36 & 1.03 & 113.40 & 70.10 & 13.98 \\
\hline HD 97671 = V602 Car & 13.50 & 10.91 & 8.39 & 3.61 & 2.49 & 1.53 & 0.99 & 0.09 & 174.90 & 85.13 & 12.40 \\
\hline V396 Cen & 12.30 & 10.00 & 7.85 & 3.58 & 2.58 & 1.56 & 1.20 & 0.79 & 53.30 & 39.81 & 4.98 \\
\hline KW Sgr & 14.81 & 12.50 & 9.66 & 4.23 & 3.12 & 1.99 & 1.54 & 0.91 & 250.10 & 148.30 & 18.39 \\
\hline 09034 = NR Vul & 15.41 & 12.41 & 9.36 & 4.49 & 3.25 & 2.14 & 1.69 & 1.00 & 106.30 & 58.80 & 12.28 \\
\hline$=$ BI Cyg & 14.83 & 12.34 & 9.33 & 3.64 & 2.35 & 1.1 & 0.58 & 0.02 & 334.60 & 244.90 & 51.23 \\
\hline KY Cyg & 17.44 & 14.63 & 11.14 & 4.01 & 2.35 & 0.85 & 0.17 & -0.46 & 510.60 & 329.60 & 50.74 \\
\hline $\mathrm{BD}+394208=\mathrm{RW}$ Cyg & 13.43 & 11.01 & 8.13 & 3.38 & 2.11 & 0.94 & 0.45 & -0.08 & 298.40 & 189.80 & 60.69 \\
\hline$\mu$ Cep & 8.88 & 6.43 & 4.17 & 0.31 & -0.52 & -1.30 & -1.65 & -2.09 & 1296.00 & 607.70 & 127.00 \\
\hline Case 75 = V 354 Cep & 16.95 & 13.85 & 10.67 & 5.20 & 3.92 & 2.57 & 1.87 & 1.39 & 77.67 & 46.56 & 8.01 \\
\hline Case $78=$ V355 Cep & 15.47 & 13.06 & 10.76 & 5.78 & 4.61 & 3.29 & 2.68 & 2.28 & 19.04 & 14.47 & 3.27 \\
\hline $\mathrm{BD}+602613=\mathrm{PZ}$ Cas & 12.84 & 11.48 & 8.90 & 3.90 & 2.42 & 1.53 & 1.02 & 0.39 & 373.00 & 398.20 & 96.48 \\
\hline $\mathrm{BD}+602634=\mathrm{TZ}$ Cas & 14.17 & 11.68 & 9.17 & 4.62 & 3.34 & 2.31 & 1.88 & 1.37 & 75.07 & 51.70 & 9.47 \\
\hline EV Car & 12.43 & 10.09 & 7.89 & 3.21 & 2.11 & 1.21 & 0.79 & 0.32 & 265.40 & 164.00 & 25.87 \\
\hline HS Cas & 14.52 & 12.33 & 9.82 & 5.13 & 3.98 & 2.88 & 2.46 & 2.05 & 23.47 & 16.41 & 3.51 \\
\hline XX Per & 11.72 & 10.36 & 8.26 & 4.25 & 3.06 & 2.21 & 1.83 & 1.19 & 70.76 & 28.42 & 4.23 \\
\hline KK Per & 12.36 & 9.97 & 7.73 & 4.30 & 3.00 & 2.14 & 1.68 & 1.78 & 19.37 & 10.87 & 2.23 \\
\hline AD Per & 12.66 & 10.13 & 7.85 & 4.30 & 3.38 & 2.48 & 1.94 & 1.65 & 21.28 & 13.90 & 2.85 \\
\hline PR Per & 12.80 & 10.14 & 7.84 & 4.60 & 3.56 & 2.68 & 2.25 & 2.14 & 12.79 & 9.39 & 2.37 \\
\hline GP Cas & 14.67 & 12.08 & 9.37 & 4.76 & 3.53 & 2.38 & 1.95 & 1.51 & 25.73 & 18.47 & 4.45 \\
\hline VY CMa & 12.01 & 10.19 & 7.95 & 3.10 & 1.96 & 0.43 & -0.73 & -2.68 & 9919.00 & 6651.00 & 1453.00 \\
\hline$\alpha$ Sco & 4.08 & 2.78 & 0.96 & -1.92 & -2.55 & -3.47 & -3.70 & -4.04 & 3198.00 & 689.90 & 115.50 \\
\hline VX Sg & 11.72 & 9.41 & 6.52 & 2.11 & 1.23 & 0.13 & -0.50 & -1.61 & 2738.00 & 1385.00 & 262.70 \\
\hline Case 49 & 17.33 & 14.26 & 11.12 & 5.41 & 4.07 & 2.74 & 2.15 & 2.01 & 17.86 & 11.01 & 6.58 \\
\hline U Lac & 12.50 & 11.04 & 8.70 & 4.30 & 2.90 & 2.13 & 1.57 & 0.77 & 124.00 & 61.49 & 9.04 \\
\hline
\end{tabular}

Table B.1. Adopted photometry for calculating $L$ and $\lambda_{\mathrm{m}}$. The $U B V I J H K L$ are in magnitudes, and the fluxes $f$ are in Janskys. 


\section{Appendix C: Wind expansion velocities}

Figure C. 1 shows the wind expansion velocities of several RSGs plotted versus luminosity. The Galactic RSG data are from Table 2 of this paper. The LMC RSG data are from Marshall et al. (2004, their Table 1 for luminosities and their Table 2 for expansion velocities). The error bars represent typical uncertainties on luminosity, $\pm 30 \%$ for the Galaxy and $\pm 20 \%$ for the LMC. The straight lines have the slope predicted by Habing et al. (1994) for dust-driven winds and were used in Jura's formula for red supergiants lacking a wind velocity measurement.

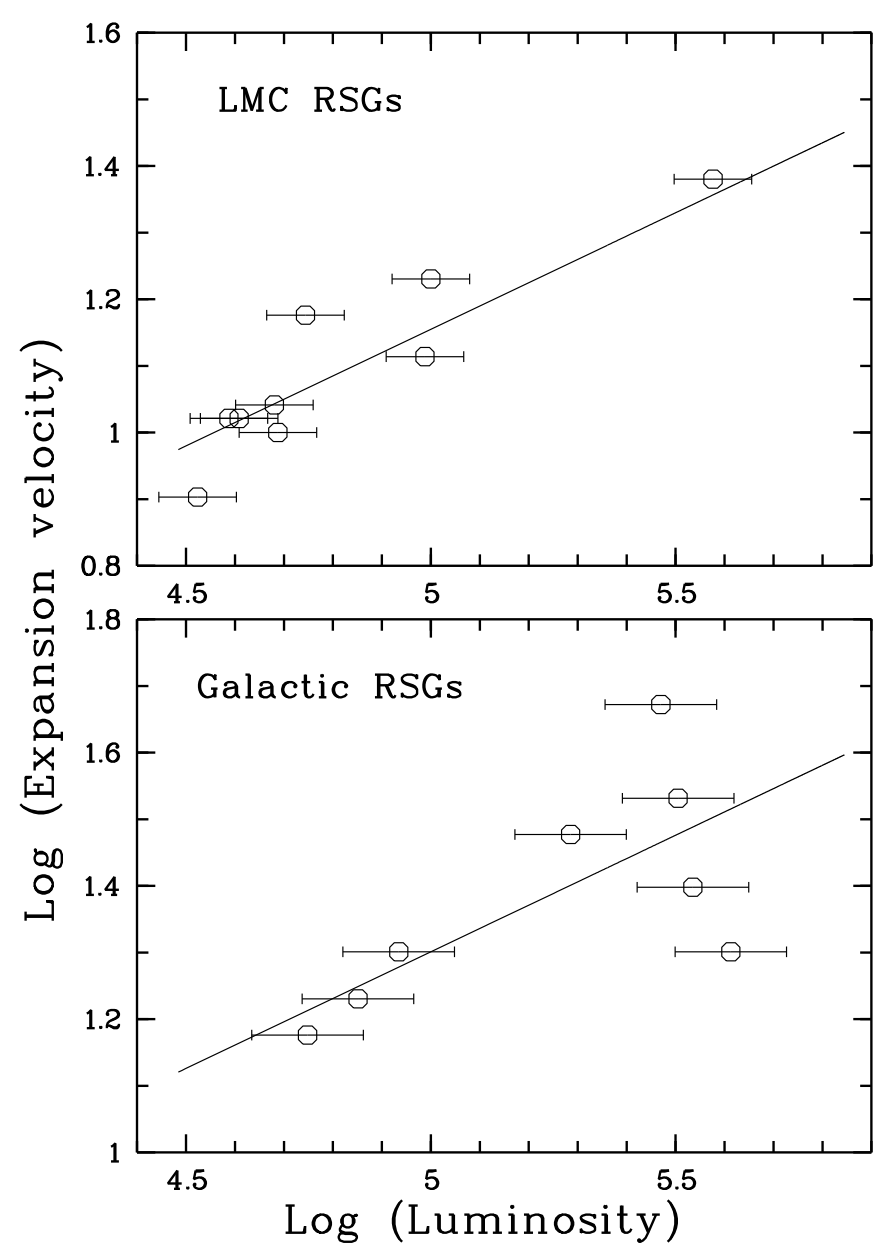

Fig. C.1. Wind expansion velocity, in $\mathrm{km} \mathrm{s}^{-1}$, as a function of luminosity (in $L_{\odot}$ ), for Galactic RSGs (lower panel), and LMC RSGs (upper panel). The straight line for Galactic RSGs corresponds to $V=20\left(L / 10^{5}\right)^{0.35} \mathrm{in} \mathrm{km} \mathrm{s}^{-1}$. The straight line for LMC RSGs corresponds to $V=14\left(L / 10^{5}\right)^{0.35}$. 